Olivier Rey · Juncheng Wei

\title{
Arbitrary number of positive solutions for an elliptic problem with critical nonlinearity
}

Received 15 April 2004 and in revised form 20 October 2004

Abstract. We show that the critical nonlinear elliptic Neumann problem

$$
\Delta u-\mu u+u^{7 / 3}=0 \quad \text { in } \Omega, \quad u>0 \quad \text { in } \Omega, \quad \frac{\partial u}{\partial v}=0 \quad \text { on } \partial \Omega,
$$

where $\Omega$ is a bounded and smooth domain in $\mathbb{R}^{5}$, has arbitrarily many solutions, provided that $\mu>0$ is small enough. More precisely, for any positive integer $K$, there exists $\mu_{K}>0$ such that for $0<\mu<\mu_{K}$, the above problem has a nontrivial solution which blows up at $K$ interior points in $\Omega$, as $\mu \rightarrow 0$. The location of the blow-up points is related to the domain geometry. The solutions are obtained as critical points of some finite-dimensional reduced energy functional. No assumption on the symmetry, geometry nor topology of the domain is needed.

Keywords. Semilinear elliptic Neumann problems, critical Sobolev exponent, blow-up

\section{Introduction}

Lin and $\mathrm{Ni}[28]$ considered the following nonlinear elliptic equation:

$$
\Delta u-\mu u+u^{q}=0 \quad \text { on } \Omega, \quad u>0 \quad \text { in } \Omega, \quad \frac{\partial u}{\partial v}=0 \quad \text { on } \partial \Omega,
$$

where $\Omega \subset \mathbb{R}^{N}(N \geq 3)$ is a smooth bounded domain, $\mu>0$ and $1<q \leq(N+2) /(N-2)$ are parameters. Such problems arise in mathematical models of chemotaxis [29] and biological pattern formation [17], [32].

The situation is known to depend highly on the parameter $\mu$. Ni and Takagi showed that for $\mu$ large enough and $1<q<(N+2) /(N-2)$, i.e. in the subcritical case, a nontrivial least energy solution exists, which concentrates at a boundary point maximizing the mean curvature of the frontier [34], [35] as $\mu$ goes to infinity. Higher energy solutions also exist, which concentrate at one or several points, located on the boundary [7], [37],

O. Rey: Centre de Mathématiques de l'École Polytechnique, 91128 Palaiseau Cedex, France; e-mail: rey@math.polytechnique.fr

J. Wei: Department of Mathematics, Chinese University of Hong Kong, Shatin, Hong Kong; e-mail: wei@math.cuhk.edu.hk

Mathematics Subject Classification (2000): Primary 35B40, 35B45; Secondary 35J40 
[13], [20], [24], [26], [49], [50], in the interior of the domain [8], [12], [14], [18], [19], [22], [48], or some of them on the boundary and others in the interior [23].

Many works have also been devoted to the critical case, i.e. $q=(N+2) /(N-2)$. As in the subcritical case, nonconstant solutions exist for $\mu$ large enough [1], [43], and the least energy solution blows up, as $\mu$ goes to infinity, at a unique point which maximizes the mean curvature of the boundary [3], [33]. Higher energy solutions have also been exhibited, blowing up at one [2], [44], [39], [21] or several (separated) boundary points [15], [30], [45], [46]. The question of interior blow-up is still open. However, in contrast with the subcritical situation, at least one blow-up point has to lie on the boundary [16], [40]. Some a priori estimates for those solutions are given in [21], [27].

In the case of small $\mu$, Lin, Ni and Takagi [29] proved in the subcritical case that problem 1.1 admits only the trivial solution (i.e., $\left.u \equiv \mu^{1 /(p-1)}\right)$. Based on this, Lin and Ni [28] asked:

Lin-Ni's Conjecture. For $\mu$ small and $q=(N+2) /(N-2)$, problem 1.1 admits only the constant solution.

The above conjecture was studied by Adimurthi-Yadava [4], [5] and Budd-KnappPeletier [10] in the case $\Omega=B_{R}(0)$ and $u$ radial. Namely, they considered the following problem:

$$
\left\{\begin{array}{l}
\Delta u-\mu u+u^{(N+2) /(N-2)}=0 \quad \text { in } B_{R}(0), \quad u>0 \quad \text { in } B_{R}(0), \\
u \text { is radial, } \quad \frac{\partial u}{\partial v}=0 \quad \text { on } \partial B_{R}(0) .
\end{array}\right.
$$

The following results were proved:

Theorem A ([4], [5], [6], [10]). For $\mu$ sufficiently small

(1) if $N=3$ or $N \geq 7$, problem (1.2) admits only the constant solution;

(2) if $N=4,5$ or 6 , problem (1.2) admits a nonconstant solution.

Theorem A reveals that Lin-Ni's conjecture depends very sensitively on the dimension $N$. A natural question is: what about general domains? (For Dirichlet boundary conditions, Brezis and Nirenberg proved that a qualitative difference occurs between $N=3$ and $N \geq 4$ [9].) The proofs of Theorem A use radial symmetry to reduce the problem to an ODE boundary value problem. Consequently, they do not carry over to general domains. In the general three-dimensional domain case, M. Zhu [52] proved:

Theorem $\mathbf{B}([52],[51])$. The conjecture is true if $N=3(q=5)$ and $\Omega$ is convex.

Zhu's proof relies strongly on a priori estimates. Recently, Wei and Xu [51] gave a direct proof of Theorem B, using only integration by parts.

The purpose of this paper is to establish a result similar to (2) of Theorem A in general five-dimensional domains, with important additional information about multiplicity and shape of solutions. Namely, we consider the problem

$$
\Delta u-\mu u+u^{7 / 3}=0 \quad \text { in } \Omega, \quad u>0 \quad \text { in } \Omega, \quad \frac{\partial u}{\partial v}=0 \quad \text { on } \partial \Omega,
$$


where $\Omega$ is a bounded and smooth domain in $\mathbb{R}^{5}$ and $\mu>0$ is small. Our main result can be stated as follows:

Main Theorem. For any integer $K \in \mathbb{N}^{*}$, there exists $\mu_{K}$ such that for $0<\mu<\mu_{K}$, problem $(1.3)$ has a solution $u_{\mu}$ which blows up at exactly $K$ interior points in $\Omega$. As a consequence, for $\mu$ small, problem (1.3) has an arbitrary number of nonconstant distinct positive solutions.

In order to make this statement more precise, some notations have to be introduced. Let $G(x, Q)$ be the Green's function defined as

$$
\Delta_{x} G(x, Q)+\delta_{Q}-\frac{1}{|\Omega|}=0 \quad \text { in } \Omega, \quad \frac{\partial G}{\partial v}=0 \quad \text { on } \partial \Omega, \quad \int_{\Omega} G(x, Q) d x=0 .
$$

We decompose

$$
G(x, Q)=K(|x-Q|)-H(x, Q),
$$

where

$$
K(r)=\frac{1}{c_{5} r^{3}}, \quad c_{5}=3\left|S^{4}\right|,
$$

is the fundamental solution of the Laplacian operator in $\mathbb{R}^{5}\left(\left|S^{4}\right|\right.$ denotes the area of the unit sphere).

For $\delta>0$ sufficiently small, we define a configuration space as

$$
\mathcal{M}_{\delta}:=\left\{\mathbf{Q}=\left(Q_{1}, \ldots, Q_{K}\right) \in \Omega^{K}\left|\min _{i} d\left(Q_{j}, \partial \Omega\right)>\delta, \min _{i \neq j}\right| Q_{i}-Q_{j} \mid>\delta\right\} .
$$

Let $\mathbf{Q}=\left(Q_{1}, \ldots, Q_{K}\right) \in \mathcal{M}_{\delta}$. We set

$$
F(\mathbf{Q})=\sum_{j=1}^{K} H\left(Q_{j}, Q_{j}\right)-\sum_{i \neq j} G\left(Q_{i}, Q_{j}\right)-K F_{0} \sum_{j=1}^{K} \int_{\Omega} \frac{1}{\left|x-Q_{j}\right|^{3}} d x
$$

where $F_{0}>0$ is a constant which depends on $\Omega$ only.

For normalization reasons, we consider throughout the paper the following equation:

$$
\Delta u-\mu u+15 u^{7 / 3}=0, \quad u>0 \quad \text { in } \Omega, \quad \frac{\partial u}{\partial v}=0 \quad \text { on } \partial \Omega
$$

instead of the original one. The solutions are identical, up to the multiplicative constant $15^{-3 / 4}$. We recall that, according to [11], the functions

$$
U_{\varepsilon, Q}(x)=\frac{\varepsilon^{3 / 2}}{\left(\varepsilon^{2}+|x-Q|^{2}\right)^{3 / 2}}, \quad \varepsilon>0, Q \in \mathbb{R}^{5},
$$

are the only solutions to the problem

$$
-\Delta u=15 u^{7 / 3}, \quad u>0 \quad \text { in } \mathbb{R}^{5} .
$$

Our main result can be stated precisely as follows: 
Theorem 1.1. Let $\Omega$ be any smooth and bounded domain in $\mathbb{R}^{5}$, and $K \in \mathbb{N}^{*}$. There exists $\mu_{K}>0$ such that for $0<\mu<\mu_{K}$, problem (1.8) has a nontrivial solution $u_{\mu}$ with the following properties:

(1) $u_{\mu}$ has $K$ local maximum points $Q_{i}^{\mu}, i=1, \ldots, K$, such that

$$
F\left(Q_{1}^{\mu}, \ldots, Q_{K}^{\mu}\right) \rightarrow \max _{\mathbf{Q} \in \mathcal{M}_{\delta}} F(\mathbf{Q}) \quad \text { as } \mu \rightarrow 0
$$

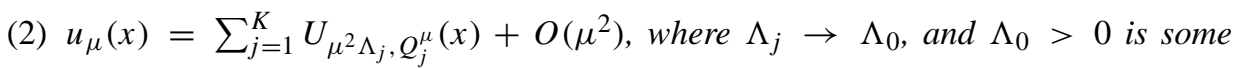
generic constant. As a consequence, $u_{\mu}\left(Q_{j}^{\mu}\right) \sim \mu^{-3}$ and $u_{\mu}(x) \rightarrow 0$ for any $x \in$ $\Omega \backslash \bigcup_{i=1}^{K} B_{\delta}\left(Q_{i}^{\mu}\right)$, where $\delta>0$ is any small number, and $u_{\mu}$ blows up at $K$ points $Q_{1}, \ldots, Q_{K}$ in $\Omega$ such that $\mathbf{Q}=\left(Q_{1}, \ldots, Q_{K}\right)$ maximizes $F$ in $\mathcal{M}_{\delta}$.

Remarks. 1. The existence of a global maximum for the function $F(\mathbf{Q})$ in $\mathcal{M}_{\delta}$ follows from the properties of the Green's function-see the proof of Lemma 6.1.

2. We believe that Theorem 1.1 should also be true in dimensions $N=4$ and $N=6$. When $N=4$, our computations show that the blow-up rate should be $e^{c_{1} / \mu^{2}}$ for some $c_{1}>0$ (instead of $\mu^{-3}$ here). When $N=6$, the blow-up rate should be $\mu^{-2}$. In both cases, the blow-up rate also depends on the location of the blow-up points. We shall come back to this question in a future work.

3. There have been many works on the multiplicity of solutions for elliptic equations with critical nonlinearity-see [31], [30], [44], [45], [46] and references therein. As far as the authors know, all the multiplicity results are proved with some additional assumptions either on the symmetry, geometry, or topology of the domain. In Theorem 1.1, no condition is required.

As we commented earlier, PDE methods have to be used to prove Theorem 1.1. Note that the least energy solution has to be constant if $\mu$ is small (see [52] and [29]). Therefore, the solutions in Theorem 1.1 must have higher energy. To capture such solutions, we use the so-called "localized energy method", a combination of the Lyapunov-Schmidt reduction method and variational techniques. Namely, we first use the Lyapunov-Schmidt method to reduce the problem to a finite-dimensional one, with some reduced energy. Then the solutions in Theorem 1.1 turn out to be generated by critical points of the reduced energy functional. This idea has been used in [22] to study the interior spike solutions of problem (1.1) when $\mu$ is large and $q$ is subcritical. This kind of argument has been applied in many other papers (see [12], [36], [19], [22], [24], [41], [42] and references therein). However, a new functional setting has to be introduced, and an appropriate variational argument to be developed to make the approach followed in our earlier works [41], [42] successful.

We set

$$
\varepsilon=\mu^{2}, \quad \Omega_{\varepsilon}:=\Omega / \varepsilon=\{z \mid \varepsilon z \in \Omega\} .
$$

Through the transformation $u(x) \mapsto \varepsilon^{-3 / 2} u(x / \varepsilon), 1.8$ becomes the rescaled problem we shall work with:

$$
\Delta u-\varepsilon^{5 / 2} u+15 u^{7 / 3}=0, \quad u>0 \quad \text { in } \Omega_{\varepsilon}, \quad \frac{\partial u}{\partial v}=0 \quad \text { on } \partial \Omega_{\varepsilon} .
$$


We set

$$
S_{\varepsilon}[u]:=-\Delta u+\varepsilon^{5 / 2} u-15 u_{+}^{7 / 3}, \quad u_{+}=\max (u, 0),
$$

and we introduce the following functional defined in $H^{1}\left(\Omega_{\varepsilon}\right)$ :

$$
J_{\varepsilon}[u]=\frac{1}{2} \int_{\Omega_{\varepsilon}}|\nabla u|^{2}+\frac{\varepsilon^{5 / 2}}{2} \int_{\Omega_{\varepsilon}} u^{2}-\frac{9}{2} \int_{\Omega_{\varepsilon}} u_{+}^{10 / 3}
$$

whose nontrivial critical points are solutions to $1.12\left(J_{\varepsilon}^{\prime}[u]=S_{\varepsilon}[u]\right)$.

The paper is organized as follows: In Section 3, we construct suitable approximate $K$-bubble solutions $W$, and list their properties. In Section 4 , we solve the linearized problem at $W$ in a finite-codimensional space. Then, in Section 4, we are able to solve the nonlinear problem in that space. In Section 5, we study the remaining finite-dimensional problem and solve it in Section 6, finding critical points of the reduced energy functional. The proof of two technical lemmas may be found in Appendices A and B.

Throughout the paper, the letters $C, C_{i}$ will denote various positive constants independent of $\varepsilon$ small. $\delta$ will always denote a small constant.

\section{Approximate bubble solutions}

This section is devoted to the construction of suitable approximate $K$-bubble solutions, in the neighbourhood of which solutions of Theorem 1.1 will be found.

Let $\varepsilon$ be as defined at (1.11). We consider $Q \in \Omega, \Lambda>0$ a constant, and $U_{\Lambda, Q / \varepsilon}$ as defined in (1.9). In view of $(1.10)$ and (1.9), $U_{\Lambda, Q / \varepsilon}$ provides us with a first approximate solution to (1.8) as $\varepsilon$ goes to zero (equivalently, $\mu$ goes to zero). However, because of the additional linear term $\mu u$ in $(1.8)$, such an approximation has to be improved. To this end, we consider the equation

$$
\Delta \Psi+U_{\Lambda}=0, \quad \Psi_{\Lambda}(x) \rightarrow 0 \quad \text { as }|x| \rightarrow+\infty
$$

where $U_{\Lambda}$ denotes $U_{\Lambda, 0}$. It is known that there exists a unique radially symmetric solution $\Psi_{\Lambda}$, which satisfies

$$
\Psi_{\Lambda}(x)=\frac{B}{|x|}\left(1+O\left(\frac{1}{|x|^{2}}\right)\right) \quad \text { for }|x|>1
$$

where $B=\Lambda^{3 / 2} / 2>0$. For $a \in \mathbb{R}^{5}$, we set

$$
\Psi_{\Lambda, a}(x)=\Psi_{\Lambda}(x-a) .
$$

(Note that $\partial_{\Lambda} \Psi_{\Lambda, a}=O\left(|x-a|^{-1}\right)$ and $\partial_{a_{i}} \Psi_{\Lambda, a}=O\left(|x-a|^{-2}\right)$ as $|x-a|$ goes to infinity.)

An additional correction is necessary, in order to obtain approximate solutions which satisfy the required boundary conditions. With this aim in view, we define

$$
\hat{U}_{\Lambda, Q / \varepsilon}(z)=-\Psi_{\Lambda, Q / \varepsilon}(z)-c_{5} \varepsilon^{1 / 2} \Lambda^{3 / 2} H(\varepsilon z, Q)+R_{\varepsilon, \Lambda, Q}(z) \chi(\varepsilon z)
$$


where $R_{\varepsilon, \Lambda, Q}$ is defined by $\Delta R_{\varepsilon, \Lambda, Q}-\varepsilon^{2} R_{\varepsilon, \Lambda, Q}=0$ in $\Omega_{\varepsilon}$ and

$$
\frac{\partial R_{\varepsilon, \Lambda, Q}}{\partial \nu}=\frac{\partial}{\partial \nu}\left[U_{\Lambda, Q / \varepsilon}-\varepsilon^{5 / 2} \Psi_{\Lambda, Q / \varepsilon}-c_{5} \varepsilon^{3} \Lambda^{3 / 2} H(\varepsilon z, Q)\right] \quad \text { on } \partial \Omega_{\varepsilon} .
$$

Lastly, $\chi(x)$ is a smooth cut-off function in $\Omega$ such that $\chi(x)=1$ for $d(x, \partial \Omega)<\delta / 4$ and $\chi(x)=0$ for $d(x, \partial \Omega)>\delta / 2$.

We notice that $(2.2)$, an expansion of $U_{\Lambda, Q / \varepsilon}$ and the definition of $H$ imply that the normal derivative of $R_{\varepsilon}, Q$ is of order $\varepsilon^{9 / 2}$ on the boundary of $\Omega_{\varepsilon}$, from which we deduce

$$
\left|R_{\varepsilon, \Lambda, Q}\right|+\left|\varepsilon^{-1} \nabla_{z} R_{\varepsilon, \Lambda, Q}\right|+\left|\varepsilon^{-2} \nabla_{z}^{2} R_{\varepsilon, \Lambda, Q}\right| \leq C \varepsilon^{7 / 2} .
$$

Such an estimate also holds for the derivatives of $R_{\varepsilon, \Lambda, Q}$ with respect to $\Lambda$ and $Q$. It will ensure that $R_{\varepsilon, \Lambda, Q}$ play no role in further computations, being negligible.

We are now able to define the appropriate approximate $K$-bubble solutions we are looking for. Let $\boldsymbol{\Lambda}=\left(\Lambda_{1}, \ldots, \Lambda_{K}\right)$ and $\mathbf{Q}=\left(Q_{1}, \ldots, Q_{K}\right)$ be such that

$$
1 / C_{0} \leq|\mathbf{\Lambda}| \leq C_{0}, \quad \mathbf{Q} \in \mathcal{M}_{\delta}
$$

In view of the rescaling, we write

$$
\bar{Q}_{i}=\frac{1}{\varepsilon} Q_{i}, \quad \overline{\mathbf{Q}}=\left(\bar{Q}_{1}, \ldots, \bar{Q}_{K}\right)
$$

and we define our approximate solutions as

$$
W_{\varepsilon, \Lambda, \overline{\mathbf{Q}}}:=\sum_{j=1}^{K}\left(U_{j}+\varepsilon^{5 / 2} \hat{U}_{j}\right)+\eta \varepsilon^{5 / 2}
$$

with

$$
\eta=\frac{c_{5}}{|\Omega|} \sum_{j=1}^{K} \Lambda_{j}^{3 / 2}
$$

To simplify our notations, we wrote $U_{j}$ and $\hat{U}_{j}$ instead of $U_{\Lambda_{j}, Q_{j} / \varepsilon}$ and $\hat{U}_{\Lambda_{j}, Q_{j} / \varepsilon}$. For the same reason, we shall also omit the dependence of $W$ on $\varepsilon, \boldsymbol{\Lambda}, \overline{\mathbf{Q}}$. The last term $\eta \varepsilon^{5 / 2}$ in (2.8) has been added to cancel, in the Laplacian of $W$, the Laplacian of $H$ introduced through the $\hat{U}_{j}$ 's. By construction, the normal derivative of $W$ vanishes on the boundary of $\Omega_{\varepsilon}$, and $W$ satisfies

$$
-\Delta W+\varepsilon^{5 / 2} W=15 \sum_{j=1}^{K} U_{j}^{7 / 3}+\varepsilon^{5} \sum_{j=1}^{K} \hat{U}_{j}-\varepsilon^{5 / 2} \Delta\left(R_{\varepsilon, Q \chi}(\varepsilon \cdot)\right) \quad \text { in } \Omega_{\varepsilon} .
$$

According to 2.5 , the last term occurring in that equation is dominated by $\varepsilon^{8}$.

We note that $W$ depends smoothly on $\Lambda, \overline{\mathbf{Q}}$. Setting, for $z \in \Omega_{\varepsilon}$,

$$
\langle z-\overline{\mathbf{Q}}\rangle=\min _{j=1}^{K}\left(1+\left|z-\bar{Q}_{j}\right|^{2}\right)^{1 / 2}
$$


we derive from the definition of $W$ the inequalities

$$
\begin{aligned}
|W(z)| & \leq C\left(\varepsilon^{5 / 2}+\langle z-\overline{\mathbf{Q}}\rangle^{-3}\right), \\
\left|D_{\boldsymbol{\Lambda}} W(z)\right| & \leq C\left(\varepsilon^{5 / 2}+\langle z-\overline{\mathbf{Q}}\rangle^{-3}\right)
\end{aligned}
$$

and

$$
\left|D_{\overline{\mathbf{Q}}} W(z)\right| \leq C\left(\varepsilon^{3}+\langle z-\overline{\mathbf{Q}}\rangle^{-4}\right)
$$

where $D_{\boldsymbol{\Lambda}}$ and $D_{\overline{\mathbf{Q}}}$ denote the first partial derivatives with respect to $\boldsymbol{\Lambda}=\left(\Lambda_{1}, \ldots, \Lambda_{K}\right)$ and $\overline{\mathbf{Q}}=\left(\bar{Q}_{1}, \ldots, \bar{Q}_{K}\right)$ respectively.

By our choice of $W$, we have the following error and energy estimates, proved in Appendix A.

Lemma 2.1. We have

$$
\left|S_{\varepsilon}[W](z)\right| \leq C\left(\varepsilon^{5 / 2}\langle z-\overline{\mathbf{Q}}\rangle^{-4}+\varepsilon^{5}\langle z-\overline{\mathbf{Q}}\rangle^{-1 / 2}\right) .
$$

The same estimate holds for $D_{\mathbf{\Lambda}} S_{\varepsilon}[W](z)$ and $D_{\overline{\mathbf{Q}}} S_{\varepsilon}[W](z)$, and

$$
\begin{aligned}
J_{\varepsilon}[W]= & A_{0}+\varepsilon^{5 / 2} \beta(\boldsymbol{\Lambda})+\varepsilon^{3} E_{0}\left[\sum_{j=1}^{K} \Lambda_{j}^{3} H\left(Q_{j}, Q_{j}\right)-\sum_{i \neq j} \Lambda_{i}^{3 / 2} \Lambda_{j}^{3 / 2} G\left(Q_{i}, Q_{j}\right)\right. \\
& \left.-F_{0}\left(\sum_{j=1}^{K} \Lambda_{j}^{3 / 2}\right) \sum_{j=1}^{K} \Lambda_{j}^{3 / 2} \int_{\Omega} \frac{d x}{\left|x-Q_{j}\right|^{3}}\right]+o\left(\varepsilon^{3}\right) .
\end{aligned}
$$

Moreover

$$
D_{\boldsymbol{\Lambda}}\left(J_{\varepsilon}[W]\right)=\varepsilon^{5 / 2} D_{\boldsymbol{\Lambda}} \beta(\boldsymbol{\Lambda})+O\left(\varepsilon^{3}\right)
$$

where $\beta(\mathbf{\Lambda})$ is defined by

$$
\beta(\boldsymbol{\Lambda})=-B_{0}\left(\sum_{j=1}^{K} \Lambda_{j}^{3 / 2}\right)^{2}+D_{0} \sum_{j=1}^{K} \Lambda_{j}^{2} .
$$

$A_{0}, B_{0}, D_{0}, E_{0}, F_{0}$ are all generic strictly positive constants.

\section{Finite-dimensional reduction: a linear problem}

According to our general strategy, we first consider the linearized problem at $W$, and we solve it in a finite-codimensional subspace, i.e. the orthogonal space to the finitedimensional subspace generated by the derivatives of $W$ with respect to the parameters $\Lambda_{j}$ and $\bar{Q}_{j, i}$. Namely, we equip $H^{1}\left(\Omega_{\varepsilon}\right)$ with the scalar product

$$
(u, v)_{\varepsilon}=\int_{\Omega_{\varepsilon}}\left(\nabla u \cdot \nabla v+\varepsilon^{5 / 2} u v\right) .
$$


Orthogonality to the functions

$$
Y_{j, 0}=\frac{\partial W}{\partial \Lambda_{j}}, \quad j=1, \ldots, K, \quad Y_{j, i}=\frac{\partial W}{\partial \bar{Q}_{j, i}}, \quad 1 \leq i \leq 5, j=1, \ldots, K,
$$

in that space is equivalent to the orthogonality in $L^{2}\left(\Omega_{\varepsilon}\right)$, equipped with the usual scalar product $\langle\cdot, \cdot\rangle$, to the functions $Z_{j, i}, 1 \leq j \leq K, 0 \leq i \leq 5$, defined as

$$
\left\{\begin{array}{l}
Z_{j, 0}=-\Delta \frac{\partial W}{\partial \Lambda_{j}}+\varepsilon^{5 / 2} \frac{\partial W}{\partial \Lambda_{j}}, \\
Z_{j, i}=-\Delta \frac{\partial W}{\partial \bar{Q}_{j, i}}+\varepsilon^{5 / 2} \frac{\partial W}{\partial \bar{Q}_{j, i}}, \quad 1 \leq i \leq 5, j=1, \ldots, K .
\end{array}\right.
$$

Note that differentiating 2.10 with respect to $\Lambda_{j}$ and $\bar{Q}_{j, i}$ and straightforward computations provide us with the estimate

$$
\left|Z_{j, i}(z)\right| \leq C\left(\varepsilon^{11 / 2}+\langle z-\overline{\mathbf{Q}}\rangle^{-7}\right) .
$$

Now, we consider the following problem: given $h$, find a function $\phi$ which satisfies

$$
\begin{cases}-\Delta \phi+\varepsilon^{5 / 2} \phi-35 W_{+}^{4 / 3} \phi=h+\sum_{j, i} c_{j, i} Z_{j, i} & \text { in } \Omega_{\varepsilon}, \\ \partial \phi / \partial \nu=0 & \text { on } \partial \Omega_{\varepsilon}, \\ \left\langle Z_{j, i}, \phi\right\rangle=0, & 0 \leq i \leq 5,1 \leq j \leq K,\end{cases}
$$

for some numbers $c_{j, i}$.

Existence and uniqueness of $\phi$ will follow from an inversion procedure in suitable function spaces. Just as del Pino, Felmer and Musso in [36], we use weighted Hölder spaces, defining here (among other possible choices) the two norms:

$$
\|\phi\|_{*}=\left\|\langle z-\overline{\mathbf{Q}}\rangle^{3 / 2} \phi(z)\right\|_{\infty}, \quad\|f\|_{* *}=\varepsilon^{-4}|\bar{f}|+\left\|\langle z-\overline{\mathbf{Q}}\rangle^{7 / 2} f(z)\right\|_{\infty},
$$

where $\|f\|_{\infty}=\max _{z \in \Omega_{\varepsilon}}|f(z)|$ and $\bar{f}=\left|\Omega_{\varepsilon}\right|^{-1} \int_{\Omega_{\varepsilon}} f(z) d z$ denotes the average of $f$ in $\Omega_{\varepsilon}$.

Before stating an existence result for $\phi$, we need the following lemma, whose proof is given in Appendix B:

Lemma 3.1. Let $u$ and $f$ satisfy

$$
-\Delta u=f \quad \text { in } \Omega_{\varepsilon}, \quad \frac{\partial u}{\partial v}=0 \quad \text { on } \partial \Omega_{\varepsilon}, \quad \bar{u}=\bar{f}=0 .
$$

Then

$$
|u(x)| \leq C \int_{\Omega_{\varepsilon}} \frac{|f(y)|}{|x-y|^{3}} d y .
$$

As a corollary, we have: 
Corollary 3.1. Suppose $u$ and $f$ satisfy

$$
-\Delta u+\varepsilon^{5 / 2} u=f \quad \text { in } \Omega_{\varepsilon}, \quad \frac{\partial u}{\partial v}=0 \quad \text { on } \partial \Omega_{\varepsilon} .
$$

Then

$$
\|u\|_{*} \leq C\|f\|_{* *} .
$$

Proof. Integrating the equation yields $\bar{u}=\varepsilon^{-5 / 2} \bar{f}$. We may write

$$
\Delta(u-\bar{u})=\varepsilon^{5 / 2}(u-\bar{u})-(f-\bar{f}) .
$$

Lemma 3.1 gives

$$
|u(y)-\bar{u}| \leq C \varepsilon^{5 / 2} \int_{\Omega_{\varepsilon}} \frac{|u(x)-\bar{u}|}{|x-y|^{3}} d x+C \int_{\Omega_{\varepsilon}} \frac{|f(x)-\bar{f}|}{|x-y|^{3}} d x .
$$

Since

$$
\langle y-\overline{\mathbf{Q}}\rangle^{3 / 2} \int_{\mathbb{R}^{5}} \frac{1}{|x-y|^{3}}\langle x-\overline{\mathbf{Q}}\rangle^{-7 / 2} d x<\infty
$$

we obtain

$$
\begin{aligned}
\left\|\langle y-\overline{\mathbf{Q}}\rangle^{3 / 2}|u-\bar{u}|\right\|_{\infty} & \leq C \varepsilon^{5 / 2}\left\|\langle y-\overline{\mathbf{Q}}\rangle^{7 / 2}|u-\bar{u}|\right\|_{\infty}+C\left\|\langle y-\overline{\mathbf{Q}}\rangle^{7 / 2}(f-\bar{f})\right\|_{\infty} \\
& \leq C \varepsilon^{1 / 2}\left\|\langle y-\overline{\mathbf{Q}}\rangle^{3 / 2} \mid u-\bar{u}\right\|_{\infty}+C\left\|\langle y-\overline{\mathbf{Q}}\rangle^{7 / 2}(f-\bar{f})\right\|_{\infty},
\end{aligned}
$$

which gives

$$
\left\|\langle y-\overline{\mathbf{Q}}\rangle^{3 / 2}|u-\bar{u}|\right\|_{\infty} \leq C\left\|\langle y-\overline{\mathbf{Q}}\rangle^{7 / 2}|f-\bar{f}|\right\|_{\infty},
$$

whence

$$
\begin{aligned}
\left\|\langle y-\overline{\mathbf{Q}}\rangle^{3 / 2} u\right\|_{\infty} & \leq C\left\|\langle y-\overline{\mathbf{Q}}\rangle^{3 / 2}\right\|_{\infty}|\bar{u}|+C \varepsilon^{-7 / 2}|\bar{f}|+\left\|\langle y-\overline{\mathbf{Q}}\rangle^{7 / 2} f\right\|_{\infty} \\
& \leq C\|f\|_{* *} .
\end{aligned}
$$

We now state the main result of this section:

Proposition 3.1. There exists $\varepsilon_{0}>0$ and a constant $C>0$, independent of $\varepsilon, \boldsymbol{\Lambda}$ and $\overline{\mathbf{Q}}$ satisfying (2.6), such that for all $0<\varepsilon<\varepsilon_{0}$ and all $h \in L^{\infty}\left(\Omega_{\varepsilon}\right)$, problem (3.4) has a unique solution $\phi \equiv L_{\varepsilon}(h)$. Furthermore

$$
\left\|L_{\varepsilon}(h)\right\|_{*} \leq C\|h\|_{* *}, \quad\left|c_{j, i}\right| \leq C\|h\|_{* *} .
$$

Moreover, the map $L_{\varepsilon}(h)$ is $C^{1}$ with respect to $\mathbf{\Lambda}, \overline{\mathbf{Q}}$ and the $L_{*}^{\infty}$-norm, and

$$
\left\|D_{(\Lambda, \overline{\mathbf{Q}})} L_{\varepsilon}(h)\right\|_{*} \leq C\|h\|_{* *} .
$$

The argument follows closely the ideas in [36], [41] and [42]. We repeat it since we use different norms. The proof relies on the following result:

Lemma 3.2. Assume that $\phi_{\varepsilon}$ solves (3.4) for $h=h_{\varepsilon}$. If $\left\|h_{\varepsilon}\right\|_{* *}$ goes to zero as $\varepsilon$ goes to zero, so does $\left\|\phi_{\varepsilon}\right\|_{*}$. 
Proof. Arguing by contradiction, we may assume that $\left\|\phi_{\varepsilon}\right\|_{*}=1$. Multiplying the first equation in (3.4) by $Y_{k, l}$ and integrating in $\Omega_{\varepsilon}$, we find

$$
\sum_{j, i} c_{j, i}\left\langle Z_{j, i}, Y_{k, l}\right\rangle=\left\langle-\Delta Y_{k, l}+\varepsilon^{5 / 2} Y_{k, l}-35 W_{+}^{4 / 3} Y_{k, l}, \phi_{\varepsilon}\right\rangle-\left\langle h_{\varepsilon}, Y_{k, l}\right\rangle
$$

On the one hand we check, in view of the definition of $Z_{j, i}, Y_{k, l}$,

$$
\begin{cases}\left\langle Z_{j, 0}, Y_{j, 0}\right\rangle=\left\|Y_{j, 0}\right\|_{\varepsilon}^{2}=\gamma_{0}+o(1), & 1 \leq j \leq K, \\ \left\langle Z_{j, i}, Y_{j, i}\right\rangle=\left\|Y_{j, i}\right\|_{\varepsilon}^{2}=\gamma_{1}+o(1), & 1 \leq i \leq 5,\end{cases}
$$

where $\gamma_{0}, \gamma_{1}$ are strictly positive constants, and

$$
\left\langle Z_{j, i}, Y_{k, l}\right\rangle=o(1), \quad j \neq k, i \neq l .
$$

On the other hand, in view of the definition of $Y_{k, l}$ and $W$, straightforward computations yield

$$
\left\langle-\Delta Y_{k, l}+\varepsilon^{5 / 2} Y_{k, l}-35 W_{+}^{4 / 3} Y_{k, l}, \phi_{\varepsilon}\right\rangle=o\left(\left\|\phi_{\varepsilon}\right\|_{*}\right)
$$

and

$$
\left\langle h_{\varepsilon}, Y_{k, l}\right\rangle=O\left(\left\|h_{\varepsilon}\right\|_{* *}\right) .
$$

Consequently, inverting the quasidiagonal linear system solved by the $c_{j, i}$ 's, we find

$$
c_{j, i}=O\left(\left\|h_{\varepsilon}\right\|_{* *}\right)+o\left(\left\|\phi_{\varepsilon}\right\|_{*}\right) .
$$

In particular, $c_{j, i}=o(1)$ as $\varepsilon$ goes to zero.

Since $\left\|\phi_{\varepsilon}\right\|_{*}=1$, elliptic theory shows that along some subsequence, the functions $\phi_{\varepsilon, j}(y)=\phi_{\varepsilon}\left(y-\bar{Q}_{j}\right)$ converge uniformly in any compact subset of $\mathbb{R}^{5}$ to a nontrivial solution of

$$
-\Delta \phi_{j}=35 U_{\Lambda_{j}, 0}^{4 / 3} \phi_{j}
$$

Moreover, $\left|\phi_{j}(y)\right| \leq C(1+|y|)^{-3 / 2}$. A bootstrap argument (see e.g. Proposition 2.2 of [47]) implies $\left|\phi_{j}(y)\right| \leq C(1+|y|)^{-3}$. As a consequence, $\phi_{j}$ can be written as

$$
\phi_{j}=\alpha_{0} \frac{\partial U_{\Lambda_{j}, 0}}{\partial \Lambda_{j}}+\sum_{i=1}^{5} \alpha_{i} \frac{\partial U_{\Lambda_{j}, 0}}{\partial y_{i}}
$$

(see [38]). On the other hand, the equalities $\left\langle Z_{j, i}, \phi_{\varepsilon}\right\rangle=0$ yield

$$
\begin{aligned}
\int_{\mathbb{R}^{5}}-\Delta \frac{\partial U_{\Lambda_{j}, 0}}{\partial \Lambda_{j}} \phi_{j} & =\int_{\mathbb{R}^{5}} U_{\Lambda_{j}, 0}^{4 / 3} \frac{\partial U_{\Lambda_{j}, 0}}{\partial \Lambda_{j}} \phi_{j}=0, \\
\int_{\mathbb{R}^{5}}-\Delta \frac{\partial U_{\Lambda_{j}, 0}}{\partial y_{i}} \phi_{j} & =\int_{\mathbb{R}^{5}} U_{\Lambda_{j}, 0}^{4 / 3} \frac{\partial U_{\Lambda_{j}, 0}}{\partial y_{i}} \phi_{j}=0, \quad 1 \leq i \leq 5 .
\end{aligned}
$$

As we also have

$$
\int_{\mathbb{R}^{5}}\left|\nabla \frac{\partial U_{\Lambda_{j}, 0}}{\partial \Lambda_{j}}\right|^{2}=\gamma_{0}>0, \quad \int_{\mathbb{R}^{5}}\left|\nabla \frac{\partial U_{\Lambda_{j}, 0}}{\partial y_{i}}\right|^{2}=\gamma_{1}>0, \quad 1 \leq i \leq 5,
$$


and

$$
\int_{\mathbb{R}^{5}} \nabla \frac{\partial U_{\Lambda_{j}, 0}}{\partial \Lambda_{j}} \cdot \nabla \frac{\partial U_{\Lambda_{j}, 0}}{\partial y_{i}}=\int_{\mathbb{R}^{5}} \nabla \frac{\partial U_{\Lambda_{j}, 0}}{\partial y_{i^{\prime}}} \cdot \nabla \frac{\partial U_{\Lambda_{j}, 0}}{\partial y_{i}}=0, \quad i \neq i^{\prime},
$$

the $\alpha_{i}$ 's solve a homogeneous quasidiagonal linear system, yielding $\alpha_{i}=0,0 \leq i \leq N$, and $\phi_{j}=0$. So $\phi_{\varepsilon}\left(z-\bar{Q}_{j}\right) \rightarrow 0$ in $C_{\text {loc }}^{1}\left(\Omega_{\varepsilon}\right)$.

Now, we remark that Corollary 3.1 provides us with the inequality

$$
\left\|\phi_{\varepsilon}\right\|_{*} \leq C\left\|W_{+}^{4 / 3} \phi_{\varepsilon}\right\|_{* *}+C\left\|h_{\varepsilon}\right\|_{* *}+C \sum_{j, i}\left|c_{j, i}\right|\left\|Z_{j, i}\right\|_{* *} .
$$

Let us estimate the right hand side. We deduce from 2.11 that

$$
\left|\langle z-\overline{\mathbf{Q}}\rangle^{7 / 2} W_{+}^{4 / 3} \phi_{\varepsilon}\right| \leq C \varepsilon^{10 / 3}\langle z-\overline{\mathbf{Q}}\rangle^{2}\left\|\phi_{\varepsilon}\right\|_{*}+C\langle z-\overline{\mathbf{Q}}\rangle^{-1 / 2}\left|\phi_{\varepsilon}\right| .
$$

Since $\left\|\phi_{\varepsilon}\right\|_{*}=1$, the first term on the right hand side is dominated by $\varepsilon^{4 / 3}$. The last term goes uniformly to zero in any ball $B_{R}\left(\bar{Q}_{j}\right)$, and is also dominated by $\langle z-\overline{\mathbf{Q}}\rangle^{-2}\left\|\phi_{\varepsilon}\right\|_{*}$ $=\langle z-\overline{\mathbf{Q}}\rangle^{-2}$, which, through the choice of $R$, can be made as small as desired in $\Omega_{\varepsilon} \backslash \bigcup_{j} B_{R}\left(\bar{Q}_{j}\right)$. Consequently,

$$
\left|\langle z-\overline{\mathbf{Q}}\rangle^{7 / 2} W_{+}^{4 / 3} \phi_{\varepsilon}\right|=o(1)
$$

as $\varepsilon$ goes to zero, uniformly in $\Omega_{\varepsilon}$. 2.11) also yields

$$
\begin{aligned}
\varepsilon^{-4} \overline{W_{+}^{4 / 3} \phi_{\varepsilon}} & \leq C \varepsilon \int_{\Omega_{\varepsilon}}\left(\varepsilon^{10 / 3}+\langle z-\overline{\mathbf{Q}}\rangle^{-4}\right)\left|\phi_{\varepsilon}\right| \\
& \left.\leq \varepsilon \int_{\Omega_{\varepsilon}}\left(\varepsilon^{10 / 3}\langle z-\overline{\mathbf{Q}}\rangle^{-3 / 2}\right)+\langle z-\overline{\mathbf{Q}}\rangle^{-11 / 2}\right)\left\|\phi_{\varepsilon}\right\|_{*} \leq \varepsilon^{5 / 6}
\end{aligned}
$$

Finally, we obtain

$$
\left\|W_{+}^{4 / 3} \phi_{\varepsilon}\right\|_{* *}=o(1)
$$

At the same time, 3.3 yields

$$
\langle z-\overline{\mathbf{Q}}\rangle^{7 / 2}\left|Z_{j, i}\right| \leq C\left(\varepsilon^{11 / 2}\langle z-\overline{\mathbf{Q}}\rangle^{7 / 2}+\langle z-\overline{\mathbf{Q}}\rangle^{-7 / 2}\right)=O(1)
$$

and

$$
\varepsilon^{-4} \overline{Z_{j, i}} \leq \varepsilon \int_{\Omega_{\varepsilon}}\left(\varepsilon^{11 / 2}\langle z-\overline{\mathbf{Q}}\rangle^{-1}+\langle z-\overline{\mathbf{Q}}\rangle^{-7}\right)=O(\varepsilon) .
$$

Then, coming back to 3.13, we find $\left\|\phi_{\varepsilon}\right\|_{*}=o(1)$ contrary to the assumption that $\left\|\phi_{\varepsilon}\right\|_{*}=1$.

Proof of Proposition 3.1. We set

$$
H=\left\{\phi \in H^{1}\left(\Omega_{\varepsilon}\right) \mid\left\langle Z_{j, i}, \phi\right\rangle=0,0 \leq i \leq 5,1 \leq j \leq K\right\},
$$

equipped with the scalar product $(\cdot, \cdot)_{\varepsilon}$. Problem (3.4) is equivalent to finding $\phi \in H$ such that

$$
(\phi, \theta)_{\varepsilon}=\left\langle 35 W_{+}^{4 / 3} \phi+h, \theta\right\rangle \quad \forall \theta \in H,
$$


that is,

$$
\phi=T_{\varepsilon}(\phi)+\tilde{h}
$$

$\tilde{h}$ depending linearly on $h$, and $T_{\varepsilon}$ being a compact operator in $H$. Fredholm's alternative ensures the existence of a unique solution, provided that the kernel of $\mathrm{Id}-T_{\varepsilon}$ is reduced to 0 . We notice that any $\phi_{\varepsilon} \in \operatorname{Ker}\left(\operatorname{Id}-T_{\varepsilon}\right)$ solves $(3.4)$ with $h=0$. Thus, we deduce from Lemma 3.2 that $\left\|\phi_{\varepsilon}\right\|_{*}=o(1)$ as $\varepsilon$ goes to zero. As $\operatorname{Ker}\left(\mathrm{Id}-T_{\varepsilon}\right)$ is a vector space, it is $\{0\}$. The inequalities 3.8 follow from Lemma 3.2 and 3.12). This completes the proof of the first part of Proposition 3.1 .

The smoothness of $L_{\varepsilon}$ with respect to $\boldsymbol{\Lambda}$ and $\overline{\mathbf{Q}}$ is a consequence of the smoothness of $T_{\varepsilon}$ and $\tilde{h}$, which occur in the implicit definition 3.14 of $\phi \equiv L_{\varepsilon}(h)$, with respect to these variables. Inequality (3.9) is obtained by differentiating (3.4), writing the derivatives of $\phi$ with respect $\boldsymbol{\Lambda}$ and $\mathbf{Q}$ as linear combinations of the $Z_{i}$ 's and an orthogonal part, and estimating each term using the first part of the proposition-see [36], [25] for detailed computations.

\section{Finite-dimensional reduction: a nonlinear problem}

In this section, we turn our attention to the nonlinear problem, which we solve in the finite-codimensional subspace orthogonal to the $Z_{j, i}$ 's. Let $S_{\varepsilon}[u]$ be as defined at $(1.13)$. Then 1.12 is equivalent to

$$
S_{\varepsilon}[u]=0 \quad \text { in } \partial \Omega_{\varepsilon}, \quad u_{+} \not \equiv 0, \quad \frac{\partial u}{\partial v}=0 \quad \text { on } \partial \Omega_{\varepsilon} .
$$

Indeed, if $u$ satisfies (4.1) the Maximum Principle ensures that $u>0$ in $\Omega_{\varepsilon}$ and (1.12) is satisfied. Observe that

$$
S_{\varepsilon}[W+\phi]=-\Delta(W+\phi)+\varepsilon^{5 / 2}(W+\phi)-15(W+\phi)_{+}^{7 / 3}
$$

may be written as

$$
S_{\varepsilon}[W+\phi]=-\Delta \phi+\varepsilon^{5 / 2} \phi-35 W_{+}^{4 / 3} \phi+R^{\varepsilon}-15 N_{\varepsilon}(\phi)
$$

with

$$
N_{\varepsilon}(\phi)=(W+\phi)_{+}^{7 / 3}-W^{7 / 3}-\frac{7}{3} W_{+}^{4 / 3} \phi
$$

and

$$
R^{\varepsilon}=S_{\varepsilon}[W]=-\Delta W+\varepsilon^{5 / 2} W-15 W^{7 / 3} .
$$

From Lemma 2.1 we derive estimates of $R^{\varepsilon}$ :

$$
\left\|R^{\varepsilon}\right\|_{* *}+\left\|D_{(\mathbf{\Lambda}, \overline{\mathbf{Q}})} R^{\varepsilon}\right\|_{* *} \leq \varepsilon^{3 / 2} .
$$


We now consider the following nonlinear problem: find $\phi$ such that, for some numbers $c_{j, i}$,

$$
\left\{\begin{array}{l}
-\Delta(W+\phi)+\varepsilon^{5 / 2}(W+\phi)-15(W+\phi)_{+}^{7 / 3}=\sum_{j, i} c_{j, i} Z_{j, i} \quad \text { in } \Omega_{\varepsilon}, \\
\partial \phi / \partial v=0 \text { on } \partial \Omega_{\varepsilon}, \\
\left\langle Z_{j, i}, \phi\right\rangle=0, \quad 1 \leq j \leq K, 0 \leq i \leq 5 .
\end{array}\right.
$$

The first equation in (4.6) reads

$$
-\Delta \phi+\varepsilon^{5 / 2} \phi-35 W^{4 / 3} \phi=15 N_{\varepsilon}(\phi)+R^{\varepsilon}+\sum_{j, i} c_{j, i} Z_{j, i}
$$

for some numbers $c_{j, i}$. The functional $N_{\varepsilon}$ may be estimated as follows:

Lemma 4.1. There exist $\varepsilon_{1}>0$, independent of $\boldsymbol{\Lambda}, \overline{\mathbf{Q}}$, and $C$, independent of $\varepsilon, \boldsymbol{\Lambda}, \overline{\mathbf{Q}}$, such that for $\varepsilon \leq \varepsilon_{1}$ and $\|\phi\|_{*} \leq \varepsilon$,

$$
\left\|N_{\varepsilon}(\phi)\right\|_{* *} \leq C \varepsilon^{5 / 6}\|\phi\|_{*},
$$

and for $\left\|\phi_{i}\right\|_{*} \leq 1$,

$$
\left\|N_{\varepsilon}\left(\phi_{1}\right)-N_{\varepsilon}\left(\phi_{2}\right)\right\|_{* *} \leq C \varepsilon^{5 / 6}\left\|\phi_{1}-\phi_{2}\right\|_{*} .
$$

Proof. We deduce from (4.3) that

$$
\left|N_{\varepsilon}(\phi)\right| \leq C\left(W_{+}^{1 / 3}|\phi|^{2}+|\phi|^{7 / 3}\right) .
$$

In view of 2.11, we compute

$$
\begin{gathered}
\varepsilon^{-4} \overline{W_{+}^{1 / 3}|\phi|^{2}+|\phi|^{7 / 3}} \leq C \varepsilon \int_{\Omega_{\varepsilon}}\left(\left(\varepsilon^{5 / 6}+\langle z-\overline{\mathbf{Q}}\rangle^{-1}\right)|\phi|^{2}+|\phi|^{7 / 3}\right) \\
\leq C \varepsilon \int_{\Omega_{\varepsilon}}\left(\left(\varepsilon^{5 / 6}\langle z-\overline{\mathbf{Q}}\rangle^{-3}+\langle z-\overline{\mathbf{Q}}\rangle^{-4}\right)\|\phi\|_{*}^{2}+\langle z-\overline{\mathbf{Q}}\rangle^{-7 / 2}\|\phi\|_{*}^{7 / 3}\right) \\
\leq C\left(\varepsilon^{-1 / 6}\|\phi\|_{*}^{2}+\varepsilon^{-1 / 2}\|\phi\|_{*}^{7 / 3}\right) \leq C \varepsilon^{5 / 6}\|\phi\|_{*} .
\end{gathered}
$$

On the other hand,

$$
\left\|\langle z-\overline{\mathbf{Q}}\rangle^{7 / 2}\left(W_{+}^{1 / 3}|\phi|^{2}+|\phi|^{7 / 3}\right)\right\|_{\infty} \leq C\|\phi\|_{*}^{2}
$$

and (4.8) follows. Concerning (4.9), we write

$$
N_{\varepsilon}\left(\phi_{1}\right)-N_{\varepsilon}\left(\phi_{2}\right)=\partial_{\eta} N_{\varepsilon}(\eta)\left(\phi_{1}-\phi_{2}\right)
$$

for some $\eta=x \phi_{1}+(1-x) \phi_{2}, x \in[0,1]$. From

$$
\partial_{\eta} N_{\varepsilon}(\eta)=\frac{7}{3}\left((W+\eta)_{+}^{4 / 3}-W_{+}^{4 / 3}\right)
$$

we deduce

$$
\left|\partial_{\eta} N_{\varepsilon}(\eta)\right| \leq C\left(W_{+}^{1 / 3}|\eta|+|\eta|^{4 / 3}\right)
$$

and the proof of (4.9) is similar to the previous one.

We now state the following result: 
Proposition 4.1. There exists $C$, independent of $\varepsilon$ and $\mathbf{\Lambda}, \overline{\mathbf{Q}}$ satisfying $(2.6$, such that for small \& problem 4.6 has a unique solution $\phi=\phi(\boldsymbol{\Lambda}, \overline{\mathbf{Q}}, \varepsilon)$ with

$$
\|\phi\|_{*} \leq C \varepsilon^{3 / 2} .
$$

Moreover, $(\boldsymbol{\Lambda}, \overline{\mathbf{Q}}) \mapsto \phi(\boldsymbol{\Lambda}, \overline{\mathbf{Q}}, \varepsilon)$ is $C^{1}$ with respect to the $*$-norm, and

$$
\left\|D_{(\boldsymbol{\Lambda}, \overline{\mathbf{Q}})} \phi\right\|_{*} \leq C \varepsilon^{3 / 2} .
$$

Proof. Following [36], we consider the map $A_{\varepsilon}$ from $\mathcal{F}=\left\{\phi \in H^{1}\left(\Omega_{\varepsilon}\right) \mid\|\phi\|_{*} \leq\right.$ $\left.C^{\prime} \varepsilon^{3 / 2}\right\}$ to $H^{1}\left(\Omega_{\varepsilon}\right)$ defined as

$$
A_{\varepsilon}(\phi)=L_{\varepsilon}\left(15 N_{\varepsilon}(\phi)+R^{\varepsilon}\right) .
$$

Here $C^{\prime}$ is a large number, to be determined later, and $L_{\varepsilon}$ is given by Proposition 3.1 We remark that finding a solution $\phi$ to problem 4.6 is equivalent to finding a fixed point of $A_{\varepsilon}$. On the one hand, we have for $\phi \in \mathcal{F}$, using (4.5), Proposition 3.1 and Lemma 4.1.

$$
\begin{aligned}
\left\|A_{\varepsilon}(\phi)\right\|_{*} & \leq\left\|L_{\varepsilon}\left(N_{\varepsilon}(\phi)\right)\right\|_{*}+\left\|L_{\varepsilon}\left(R^{\varepsilon}\right)\right\|_{*} \leq C_{1}\left(\left\|N_{\varepsilon}(\phi)\right\|_{* *}+\varepsilon^{3 / 2}\right) \\
& \leq C_{2} C^{\prime} \varepsilon^{3 / 2+5 / 6}+C_{1} \varepsilon^{3 / 2} \leq C^{\prime} \varepsilon^{3 / 2}
\end{aligned}
$$

for $C^{\prime}=2 C_{1}$ and $\varepsilon$ small enough, implying that $A_{\varepsilon}$ sends $\mathcal{F}$ into itself. On the other hand, $A_{\varepsilon}$ is a contraction. Indeed, for $\phi_{1}$ and $\phi_{2}$ in $\mathcal{F}$, we write

$$
\left\|A_{\varepsilon}\left(\phi_{1}\right)-A_{\varepsilon}\left(\phi_{2}\right)\right\|_{*} \leq C\left\|N_{\varepsilon}\left(\phi_{1}\right)-N_{\varepsilon}\left(\phi_{2}\right)\right\|_{* *} \leq C \varepsilon^{5 / 6}\left\|\phi_{1}-\phi_{2}\right\|_{*} \leq \frac{1}{2}\left\|\phi_{1}-\phi_{2}\right\|_{*}
$$

for $\varepsilon$ small enough. The Contraction Mapping Theorem implies that $A_{\varepsilon}$ has a unique fixed point in $\mathcal{F}$, that is, problem $\left(4.6\right.$ has a unique solution $\phi$ such that $\|\phi\|_{*} \leq C^{\prime} \varepsilon^{3 / 2}$.

In order to prove that $(\boldsymbol{\Lambda}, \mathbf{Q}) \mapsto \phi(\boldsymbol{\Lambda}, \overline{\mathbf{Q}})$ is $C^{1}$, we remark that if we set for $\eta \in \mathcal{F}$,

$$
B(\boldsymbol{\Lambda}, \overline{\mathbf{Q}}, \eta) \equiv \eta-L_{\varepsilon}\left(15 N_{\varepsilon}(\eta)+R^{\varepsilon}\right)
$$

then $\phi$ is defined as

$$
B(\boldsymbol{\Lambda}, \overline{\mathbf{Q}}, \phi)=0
$$

We have

$$
\left.\partial_{\eta} B(\boldsymbol{\Lambda}, \overline{\mathbf{Q}}, \eta)[\theta]=\theta-15 L_{\varepsilon}\left(\theta \partial_{\eta} N_{\varepsilon}\right)(\eta)\right) .
$$

Using Proposition 3.1 and 4.11 we write

$$
\begin{aligned}
\left\|L_{\varepsilon}\left(\theta\left(\partial_{\eta} N_{\varepsilon}\right)(\eta)\right)\right\|_{*} & \leq C\left\|\theta\left(\partial_{\eta} N_{\varepsilon}\right)(\eta)\right\|_{* *} \leq C\left\|\langle z-\overline{\mathbf{Q}}\rangle^{-3 / 2}\left(\partial_{\eta} N_{\varepsilon}\right)(\eta)\right\|_{* *}\|\theta\|_{*} \\
& \leq C\left\|\langle z-\overline{\mathbf{Q}}\rangle^{-3 / 2}\left(\left.\left|W_{+}^{1 / 3}\right| \eta|+| \eta\right|^{4 / 3}\right)\right\|_{* *}\|\theta\|_{*} .
\end{aligned}
$$

In view of 3.5 , 2.11) and $\eta \in \mathcal{F}$, we obtain

$$
\left\|L_{\varepsilon}\left(\theta\left(\partial_{\eta} N_{\varepsilon}\right)(\eta)\right)\right\|_{*} \leq C \varepsilon^{3 / 2}\|\theta\|_{*} .
$$


Consequently, $\partial_{\eta} B(\boldsymbol{\Lambda}, \overline{\mathbf{Q}}, \phi)$ is invertible with uniformly bounded inverse. Then the fact that $(\boldsymbol{\Lambda}, \overline{\mathbf{Q}}) \mapsto \phi(\boldsymbol{\Lambda}, \overline{\mathbf{Q}})$ is $C^{1}$ follows from the fact that $(\boldsymbol{\Lambda}, \overline{\mathbf{Q}}, \eta) \mapsto L_{\varepsilon}\left(N_{\varepsilon}(\eta)\right)$ is $C^{1}$ and the implicit function theorem.

Finally, let us show how estimate (4.13) may be obtained. Differentiating (4.14) with respect to $\Lambda$, we find

$$
\partial_{\Lambda} \phi=\left(\partial_{\eta} B(\Lambda, \xi, \phi)\right)^{-1}\left(\left(\partial_{\Lambda} L_{\varepsilon}\right)\left(N_{\varepsilon}(\phi)\right)+L_{\varepsilon}\left(\left(\partial_{\Lambda} N_{\varepsilon}\right)(\phi)\right)+L_{\varepsilon}\left(\partial_{\Lambda} R^{\varepsilon}\right)\right),
$$

whence, according to Proposition 3.1 .

$$
\left\|\partial_{\boldsymbol{\Lambda}} \phi\right\|_{*} \leq C\left(\left\|N_{\varepsilon}(\phi)\right\|_{* *}+\left\|\left(\partial_{\boldsymbol{\Lambda}} N_{\varepsilon}\right)(\phi)\right\|_{* *}+\left\|\partial_{\boldsymbol{\Lambda}} R^{\varepsilon}\right\|_{* *}\right) .
$$

From Lemma 4.1 and 4.12 we know that

$$
\left\|N_{\varepsilon}(\phi)\right\|_{* *} \leq C \varepsilon^{3 / 2} .
$$

Concerning the next term, we notice that according to the definition of $N_{\varepsilon}$,

$$
\left|\left(\partial_{\Lambda} N_{\varepsilon}\right)(\phi)\right|=\frac{7}{3}\left|(W+\phi)_{+}^{4 / 3}-W_{+}^{4 / 3}-\frac{4}{3} W_{+}^{1 / 3} \phi\right|\left|\partial_{\Lambda} W\right|,
$$

whence again, using (2.11), (2.12) and (4.12),

$$
\left\|\left(\partial_{\Lambda} N_{\varepsilon}\right)(\phi)\right\|_{* *} \leq C \varepsilon^{3 / 2} .
$$

Finally, using 4.5 , we obtain

$$
\left\|\partial_{\Lambda} \phi\right\|_{*} \leq C \varepsilon^{3 / 2} .
$$

The derivative of $\phi$ with respect to $\overline{\mathbf{Q}}$ may be estimated in the same way. This concludes the proof of Proposition 4.1

\section{Finite-dimensional reduction: reduced energy}

Let us define a reduced energy functional as

$$
I_{\varepsilon}(\mathbf{\Lambda}, \mathbf{Q}) \equiv J_{\varepsilon}\left[W_{\boldsymbol{\Lambda}, \overline{\mathbf{Q}}}+\phi_{\varepsilon, \boldsymbol{\Lambda}, \overline{\mathbf{Q}}}\right] .
$$

Then we state:

Proposition 5.1. The function $u=W+\phi$ is a solution to problem (1.12) if and only if $(\boldsymbol{\Lambda}, \overline{\mathbf{Q}})$ is a critical point of $I_{\varepsilon}$.

Proof. We notice that $u=W+\phi$ being a solution to 1.12 is equivalent to being a critical point of $J_{\varepsilon}$. It is also equivalent to the cancellation of the $c_{j, i}$ 's in (4.6) or, in view of 3.10) and 3.11,

$$
J_{\varepsilon}^{\prime}[W+\phi]\left[Y_{j, i}\right]=0, \quad 1 \leq j \leq K, 0 \leq i \leq 5 .
$$


On the other hand, we deduce from 5.1 that $I_{\varepsilon}^{\prime}(\boldsymbol{\Lambda}, \mathbf{Q})=0$ is equivalent to the cancellation of $J_{\varepsilon}^{\prime}(W+\phi)$ applied to the derivatives of $W+\phi$ with respect to $\boldsymbol{\Lambda}$ and $\overline{\mathbf{Q}}$. According to the definition 3.1) of the $Y_{j, i}$ 's and Proposition 4.1 we have

$$
\frac{\partial(W+\phi)}{\partial \Lambda_{j}}=Y_{j, 0}+y_{j, 0}, \quad 1 \leq j \leq K, \quad \frac{\partial(W+\phi)}{\partial \bar{Q}_{j, i}}=Y_{j, i}+y_{j, i}, \quad 1 \leq i \leq 5,
$$

with $\left\|y_{j, i}\right\|_{*}=o(1), 1 \leq j \leq K, 0 \leq i \leq 5$. Writing

$y_{j, i}=y_{j, i}^{\prime}+\sum_{k, l} a_{j i, k l} Y_{k, l}, \quad\left\langle y_{j, i}^{\prime}, Z_{k, l}\right\rangle=\left(y_{j, i}^{\prime}, Y_{j, i}\right)_{\varepsilon}=0, \quad 0 \leq i \leq 5,1 \leq j \leq K$,

and

$$
J_{\varepsilon}^{\prime}[W+\phi]\left[Y_{j, i}\right]=\alpha_{j, i}
$$

it turns out that $I_{\varepsilon}^{\prime}(\boldsymbol{\Lambda}, \overline{\mathbf{Q}})=0$ is equivalent, since $J_{\varepsilon}^{\prime}[W+\phi][\theta]=0$ for $\left\langle\theta, Z_{j, i}\right\rangle=$ $\left(\theta, Y_{j, i}\right)_{\varepsilon}=0,1 \leq j \leq K, 0 \leq i \leq 5$, to

$$
\left(\mathrm{Id}+\left[a_{j i, k l}\right]\right)\left[\alpha_{j i}\right]=0 .
$$

As $a_{j i, k l}=O\left(\left\|y_{k, l}\right\|_{*}\right)=o(1)$, we see that $I_{\varepsilon}^{\prime}(\mathbf{\Lambda}, \mathbf{Q})=0$ means exactly that 5.2 is satisfied.

In view of Proposition 5.1, to prove the theorem, we have to find critical points of $I_{\varepsilon}$. We establish an expansion of $I_{\varepsilon}$.

Proposition 5.2. For $\varepsilon$ sufficiently small, we have

$$
I_{\varepsilon}(\boldsymbol{\Lambda}, \mathbf{Q})=J_{\varepsilon}[W]+\varepsilon^{3} \sigma_{\varepsilon}(\boldsymbol{\Lambda}, \mathbf{Q})
$$

where $\sigma_{\varepsilon}=o(1)$ and $D_{\Lambda} \sigma_{\varepsilon}=O(1)$ as $\varepsilon$ goes to zero, uniformly with respect to $\mathbf{\Lambda}, \mathbf{Q}$ satisfying (2.6.

Proof. We first prove that

$$
I_{\varepsilon}(\boldsymbol{\Lambda}, \mathbf{Q})-J_{\varepsilon}[W]=o\left(\varepsilon^{3}\right) .
$$

Actually, in view of 5.1 , a Taylor expansion and the fact that $J_{\varepsilon}^{\prime}[W+\phi][\phi]=0$ yield

$$
\begin{aligned}
I_{\varepsilon}(\boldsymbol{\Lambda}, \mathbf{Q})-J_{\varepsilon}[W] & =J_{\varepsilon}[W+\phi]-J_{\varepsilon}[W]=-\int_{0}^{1} J_{\varepsilon}^{\prime \prime}(W+t \phi)[\phi, \phi] t d t \\
& =-\int_{0}^{1}\left(\int_{\Omega_{\varepsilon}}\left(|\nabla \phi|^{2}+\varepsilon^{5 / 2} \phi^{2}-35(W+t \phi)_{+}^{4 / 3} \phi^{2}\right)\right) t d t,
\end{aligned}
$$

whence

$$
\begin{aligned}
& I_{\varepsilon}(\boldsymbol{\Lambda}, \mathbf{Q})-J_{\varepsilon}[W] \\
& =-\int_{0}^{1}\left(15 \int_{\Omega_{\varepsilon}}\left(N_{\varepsilon}(\phi) \phi+\frac{7}{3}\left[W_{+}^{4 / 3}-(W+t \phi)_{+}^{4 / 3}\right] \phi^{2}\right)\right) t d t-\int_{\Omega_{\varepsilon}} R^{\varepsilon} \phi .
\end{aligned}
$$


From 4.3, 2.11) and Proposition 4.1, we deduce that the first term on the right hand side satisfies

$$
\left|\int_{\Omega_{\varepsilon}} N_{\varepsilon}(\phi) \phi\right| \leq C \int_{\Omega_{\varepsilon}}\left(W_{+}^{1 / 3}|\phi|^{3}+|\phi|^{10 / 3}\right) \leq C \varepsilon^{4} .
$$

Similarly, for the second term on the right hand side we obtain

$$
\left|\int_{\Omega_{\varepsilon}}\left(W_{+}^{4 / 3}-(W+t \phi)_{+}^{4 / 3}\right) \phi^{2}\right| \leq C \int_{\Omega_{\varepsilon}}\left(W_{+}^{1 / 3}|\phi|^{3}+|\phi|^{10 / 3}\right) \leq C \varepsilon^{4} .
$$

Concerning the last integral, we remark that according to 2.14,

$$
\left|R^{\varepsilon}\right| \leq C \varepsilon^{5 / 2}\langle z-\overline{\mathbf{Q}}\rangle^{-4}+C \varepsilon^{5}\langle z-\overline{\mathbf{Q}}\rangle^{-1 / 2}
$$

uniformly in $\Omega_{\varepsilon}$. Therefore

$$
\left|\int_{\Omega_{\varepsilon}} R^{\varepsilon} \phi\right| \leq C\|\phi\|_{*} \int_{\Omega_{\varepsilon}} \varepsilon^{5 / 2}\langle z-\overline{\mathbf{Q}}\rangle^{-11 / 2}+\varepsilon^{5}\|\phi\|_{*} \int_{\Omega_{\varepsilon}}\langle z-\overline{\mathbf{Q}}\rangle^{-2} \leq C \varepsilon^{7 / 2} .
$$

This concludes the proof of 5.4 ).

An estimate for the derivative with respect to $\boldsymbol{\Lambda}$ is established exactly in the same way, differentiating the right hand side in 5.5 and estimating each term separately, using 4.3, 4.5 and Lemma 2.1 (see Proposition 3.4 in [25] for detailed computations).

\section{Proof of Theorem 1.1}

In view of Proposition 5.1 proving Theorem 1.1 turns out to be equivalent to proving the existence of a critical point of $I_{\varepsilon}(\boldsymbol{\Lambda}, \mathbf{Q})$. According to Proposition 5.2 and Lemma 2.1, setting

$$
K_{\varepsilon}(\boldsymbol{\Lambda}, \mathbf{Q}):=\frac{I_{\varepsilon}(\boldsymbol{\Lambda}, \mathbf{Q})-A_{0}}{\varepsilon^{5 / 2}}
$$

we have the expansion

$$
\begin{aligned}
K_{\varepsilon}(\boldsymbol{\Lambda}, \mathbf{Q})= & \beta(\boldsymbol{\Lambda})+\varepsilon^{1 / 2} E_{0}\left[\sum_{j=1}^{K} \Lambda_{j}^{3} H\left(Q_{j}, Q_{j}\right)-\sum_{i \neq j} \Lambda_{i}^{3 / 2} \Lambda_{j}^{3 / 2} G\left(Q_{i}, Q_{j}\right)\right. \\
& \left.-F_{0}\left(\sum_{j=1}^{K} \Lambda_{j}^{3 / 2}\right) \sum_{j=1}^{K} \Lambda_{j}^{3 / 2} \int_{\Omega} \frac{d x}{\left|x-Q_{j}\right|^{3}}\right]+o\left(\varepsilon^{1 / 2}\right)
\end{aligned}
$$

and

$$
D_{\boldsymbol{\Lambda}} K_{\varepsilon}(\boldsymbol{\Lambda}, \mathbf{Q})=D_{\boldsymbol{\Lambda}} \beta(\boldsymbol{\Lambda})+O\left(\varepsilon^{1 / 2}\right)
$$

with

$$
\beta(\boldsymbol{\Lambda})=-B_{0}\left(\sum_{j=1}^{K} \Lambda_{j}^{3 / 2}\right)^{2}+D_{0} \sum_{j=1}^{K} \Lambda_{j}^{2}
$$


We notice that $\beta(\boldsymbol{\Lambda}) \rightarrow-\infty$ as $|\boldsymbol{\Lambda}| \rightarrow \infty$. Except for $K=1$, the maximum points of $\beta$ in $\overline{\mathbb{R}_{+}^{K}}$ lie on the boundary of this set. However, computing the first derivatives

$$
\partial_{\Lambda_{i}} \beta(\boldsymbol{\Lambda})=-3 B_{0}\left(\sum_{j=1}^{K} \Lambda_{j}^{3 / 2}\right) \Lambda_{i}^{1 / 2}+2 D_{0} \Lambda_{i}
$$

we see that, in any case, $\beta$ has a (unique) critical point $\hat{\Lambda}_{0}$ in the interior of $\mathbb{R}_{+}^{K}$ such that

$$
\hat{\boldsymbol{\Lambda}}_{0}=\left(\Lambda_{0}, \ldots, \Lambda_{0}\right), \quad \Lambda_{0}=\frac{2 D_{0}}{3 B_{0} K}, \quad \beta\left(\hat{\Lambda}_{0}\right)=\frac{4 D_{0}^{3}}{27 B_{0}^{2} K} .
$$

We compute

$$
\partial_{\Lambda_{i} \Lambda_{j}}^{2} \beta\left(\hat{\boldsymbol{\Lambda}}_{0}\right)=D_{0}\left(-3 / K+\delta_{i j}\right) .
$$

Thus, the eigenvalues of $\beta^{\prime \prime}$ are $\lambda^{+}=D_{0}$, with multiplicity $K-1$, and $\lambda^{-}=-2 D_{0}$, with multiplicity one. Consequently, $\hat{\Lambda}_{0}$ is a maximum point in the $(1, \ldots, 1)$ direction, corresponding to $\lambda^{-}$, and a minimum point in the orthogonal hyperplane (when $K \geq 2$ ).

We also remark that for $\boldsymbol{\Lambda}=\hat{\boldsymbol{\Lambda}}_{0}$, the term in square brackets in the expansion 6.1 of $K_{\varepsilon}$ can be written as $\Lambda_{0}^{3} F(\mathbf{Q})$ with

$$
F(\mathbf{Q})=\sum_{j=1}^{K} H\left(Q_{j}, Q_{j}\right)-\sum_{i \neq j} G\left(Q_{i}, Q_{j}\right)-F_{0} K \sum_{j=1}^{K} \int_{\Omega} \frac{d x}{\left|x-Q_{j}\right|^{3}} .
$$

Note also that $F$ achieves its maximum $\hat{F}$ in the interior of $\mathcal{M}_{\delta}$. More precisely, we shall prove:

Lemma 6.1. There exists a constant $C>0$ such that

$$
\sup _{\mathbf{Q} \in \partial \mathcal{M}_{\delta}} F(\mathbf{Q}) \leq-C / \delta^{3} \quad \text { as } \delta \rightarrow 0 .
$$

Considering these facts, our aim is to prove that for $\varepsilon$ small enough, $K_{\varepsilon}$ has a critical point $(\hat{\mathbf{\Lambda}}, \hat{\mathbf{Q}})$, with $\hat{\boldsymbol{\Lambda}}$ close to $\hat{\boldsymbol{\Lambda}}_{0}$ and $\hat{\mathbf{Q}}$ close to a maximum point of $F$. In order to use a linking argument, we set

$$
\Sigma=\left\{(\boldsymbol{\Lambda}, \mathbf{Q}) \mid \mathbf{Q} \in \mathcal{M}_{\delta}, 1 / C_{0}<\Lambda_{i}<C_{0}, 1 \leq i \leq K\right\}
$$

where $C_{0}$ is a large constant. We also define a closed subset of $\Sigma$,

$$
\mathcal{B}=\left\{(\boldsymbol{\Lambda}, \mathbf{Q})|\mathbf{Q} \in \mathcal{U},| \boldsymbol{\Lambda}-\hat{\boldsymbol{\Lambda}}_{0} \mid \leq \alpha\right\}
$$

where $\mathcal{U}$ is a closed contractible neighbourhood of a maximum point of $F$, and $\alpha>0$ is a fixed small number. Lastly, we define $\mathcal{B}_{0}$, a closed subset of $\mathcal{B}$, as

$$
\mathcal{B}_{0}=\left\{(\boldsymbol{\Lambda}, \mathbf{Q})|\mathbf{Q} \in \mathcal{U},| \boldsymbol{\Lambda}-\hat{\boldsymbol{\Lambda}}_{0} \mid=\alpha,\left(\boldsymbol{\Lambda}-\hat{\boldsymbol{\Lambda}}_{0}\right) \cdot \hat{\boldsymbol{\Lambda}}_{0}=0\right\} .
$$


In view of the behaviour of $\beta$ at $\hat{\Lambda}_{0}, \alpha$ is chosen small enough so that for any $(\boldsymbol{\Lambda}, \mathbf{Q}) \in \mathcal{B}_{0}$, $\beta(\boldsymbol{\Lambda})>\beta\left(\hat{\boldsymbol{\Lambda}}_{0}\right)$. Finally, we set

$$
\Gamma=\left\{\varphi \in C^{0}(\mathcal{B}, \Sigma)|\varphi|_{\mathcal{B}_{0}}=\mathrm{Id}\right\}, \quad c=\max _{\varphi \in \Gamma} \min _{(\boldsymbol{\Lambda}, \mathbf{Q}) \in \mathcal{B}} K_{\varepsilon}(\varphi(\boldsymbol{\Lambda}, \mathbf{Q})) .
$$

We show that $c$ is a critical value of $K_{\varepsilon}$. To this end, standard deformation arguments ensure that it is sufficient to prove:

(H1) $\min _{(\boldsymbol{\Lambda}, \mathbf{Q}) \in \mathcal{B}_{0}} K_{\varepsilon}(\boldsymbol{\Lambda}, \mathbf{Q})>c$.

(H2) For all $(\boldsymbol{\Lambda}, \mathbf{Q}) \in \partial \Sigma$ such that $K_{\varepsilon}(\boldsymbol{\Lambda}, \mathbf{Q})=c$, there exists $\tau_{(\mathbf{\Lambda}, \mathbf{Q})}$, a tangent vector to $\partial \Sigma$ at $(\boldsymbol{\Lambda}, \mathbf{Q})$, such that

$$
\partial_{\tau_{(\boldsymbol{\Lambda}, \mathbf{Q})}} K_{\varepsilon}(\mathbf{\Lambda}, \mathbf{Q}) \neq 0 .
$$

Before proving (H1) and (H2), we need to estimate $c$. We remark that for any $\varphi$ in $\Gamma$, there exists some $\left(\boldsymbol{\Lambda}^{\prime}, \mathbf{Q}^{\prime}\right)=\varphi(\boldsymbol{\Lambda}, \mathbf{Q}),(\boldsymbol{\Lambda}, \mathbf{Q}) \in \mathcal{B}$, such that $\boldsymbol{\Lambda}^{\prime}$ is proportional to $(1, \ldots, 1)$. (This follows from the fact that $\varphi \in C^{0}(\mathcal{B}, \Sigma)$ and $\left.\varphi\right|_{\mathcal{B}_{0}}=$ Id.) Then, according to 6.1 and 6.5),

$$
K_{\varepsilon}\left(\boldsymbol{\Lambda}^{\prime}, \mathbf{Q}^{\prime}\right)=\beta\left(\mathbf{\Lambda}^{\prime}\right)+\varepsilon^{1 / 2} E_{0} \Lambda^{\prime 3} F\left(\mathbf{Q}^{\prime}\right)+o\left(\varepsilon^{1 / 2}\right) .
$$

Maximizing the right hand side with respect to $\boldsymbol{\Lambda}^{\prime}$ proportional to $(1, \ldots, 1)$ and $\mathbf{Q}^{\prime}$ in $\mathcal{M}_{\delta}$, we see that for any $\varphi$ in $\Gamma$, there exists some $\left(\mathbf{\Lambda}^{\prime}, \mathbf{Q}^{\prime}\right)$ such that

$$
K_{\varepsilon}\left(\mathbf{\Lambda}^{\prime}, \mathbf{Q}^{\prime}\right) \leq \beta\left(\hat{\mathbf{\Lambda}}_{0}\right)+\varepsilon^{1 / 2} E_{0} \Lambda_{0}^{3} \hat{F}+o\left(\varepsilon^{1 / 2}\right),
$$

whence also

$$
c \leq \beta\left(\hat{\Lambda}_{0}\right)+\varepsilon^{1 / 2} E_{0} \Lambda_{0}^{3} \hat{F}+o\left(\varepsilon^{1 / 2}\right) .
$$

On the other hand, we consider a special $\varphi$ such that, if we set $\left(\boldsymbol{\Lambda}^{\prime}, \mathbf{Q}^{\prime}\right)=\varphi(\boldsymbol{\Lambda}, \mathbf{Q})$ for $(\boldsymbol{\Lambda}, \mathbf{Q}) \in \mathcal{B}$, then $\boldsymbol{\Lambda}^{\prime}$ is the orthogonal projection of $\boldsymbol{\Lambda}$ over the disk $D=\left\{\boldsymbol{\Lambda}|| \boldsymbol{\Lambda}-\hat{\boldsymbol{\Lambda}}_{0} \mid\right.$ $\left.\leq \alpha,\left(\boldsymbol{\Lambda}-\hat{\boldsymbol{\Lambda}}_{0}\right) \cdot \hat{\boldsymbol{\Lambda}}_{0}=0\right\}$. Moreover, we choose $\varphi$ in such a way that, for $\left|\boldsymbol{\Lambda}-\hat{\boldsymbol{\Lambda}}_{0}\right| \leq \alpha / 2$, $\mathbf{Q}^{\prime}$ is a maximum point of $F$ (this is possible, since we assumed that $\mathcal{U}$ is a closed contractible neighbourhood of a maximum point of $F$ ). In view of 6.1 and the behaviour of $\beta$, for such $\varphi$ and $\varepsilon$ small enough we have

$$
\min _{(\boldsymbol{\Lambda}, \mathbf{Q}) \in \mathcal{B}} K_{\varepsilon}(\varphi(\boldsymbol{\Lambda}, \mathbf{Q}))=\beta\left(\hat{\boldsymbol{\Lambda}}_{0}\right)+\varepsilon^{1 / 2} E_{0} \Lambda_{0}^{\prime 3} \hat{F}+o\left(\varepsilon^{1 / 2}\right),
$$

whence the reverse inequality to (6.7), and the final estimate

$$
c=\beta\left(\hat{\Lambda}_{0}\right)+\varepsilon^{1 / 2} E_{0} \Lambda_{0}^{\prime 3} \hat{F}+o\left(\varepsilon^{1 / 2}\right) .
$$

Let us now show that $(\mathrm{H} 1)$ and $(\mathrm{H} 2)$ are satisfied. In view of 6.8$)$, the inequality in (H1) follows directly from the expansion 6.1 , the definition of $\mathcal{B}_{0}$ and the properties of $\beta$, provided that $\varepsilon$ is small enough.

We are left with the proof of $(\mathrm{H} 2)$. We note that $K_{\varepsilon}(\boldsymbol{\Lambda}, \mathbf{Q})=c$ implies, through 6.1 , that

$$
\beta(\boldsymbol{\Lambda})=c+O\left(\varepsilon^{1 / 2}\right) .
$$


As already stated, $\beta(\boldsymbol{\Lambda}) \rightarrow-\infty$ as soon as some $\Lambda_{i}$ goes to infinity. Therefore, 6.9 implies that $\Lambda_{i} \leq C_{1}, 1 \leq i \leq K$, for some constant $C_{1}$. On the other hand, suppose that $\Lambda_{i}$ goes to zero for some indices, say $1 \leq i \leq m$. If $m=K$, then $\beta(\boldsymbol{\Lambda})$ goes to zero, a contradiction with 6.9]. If $m<K$, there exists some index $j \geq m+1$ such that $\partial_{\Lambda_{j}} \beta(\boldsymbol{\Lambda}) \neq 0$. Indeed, if not, in view of 6.3 we would obtain

$$
\Lambda_{j}=\frac{2 D_{0}}{3 B_{0}(K-m)}+o(1), \quad m+1 \leq j \leq K
$$

whence

$$
\beta(\boldsymbol{\Lambda})=\frac{4 D_{0}^{3}}{27 B_{0}^{2}(K-m)}+o(1),
$$

and again, comparing with 6.4, a contradiction with 6.9). Consequently, there exists an index $j \geq m+1$ such that $\partial_{\Lambda_{j}} \beta(\boldsymbol{\Lambda}) \neq 0$, implying through $(6.2)$ that $\partial_{\Lambda_{j}} K_{\varepsilon}(\boldsymbol{\Lambda}, \mathbf{Q}) \neq 0$ for $\varepsilon$ small enough. Then we see that if we choose $C_{0}>C_{1}$ large enough in the definition of $\Sigma$, (H2) is satisfied when $(\boldsymbol{\Lambda}, \mathbf{Q}) \in \partial \Sigma$ with $K_{\varepsilon}(\boldsymbol{\Lambda}, \mathbf{Q})=c$ is such that $\Lambda_{i}=C_{0}$ (impossible) or $\Lambda_{i}=1 / C_{0}$ (taking $\tau_{(\mathbf{\Lambda}, \mathbf{Q})}=\partial_{\Lambda_{j}}$ for some appropriate index $j$ ).

It only remains to consider the case $1 / C_{0}<\Lambda_{j}<C_{0}, 1 \leq j \leq K$, and $\mathbf{Q} \in \partial \mathcal{M}_{\delta}$. If there exists some index $j$ such that $\partial_{\Lambda_{j}} K_{\varepsilon}(\boldsymbol{\Lambda}, \mathbf{Q}) \neq 0$, then (H2) holds. If not, it follows from 6.2 and 6.3 that

$$
\boldsymbol{\Lambda}=\hat{\mathbf{\Lambda}}_{0}+O\left(\varepsilon^{1 / 2}\right) \quad \text { and } \quad \beta(\boldsymbol{\Lambda})=\beta\left(\hat{\mathbf{\Lambda}}_{0}\right)+O(\varepsilon) .
$$

Thus, 6.1) yields

$$
K_{\varepsilon}(\boldsymbol{\Lambda}, \mathbf{Q})=\beta\left(\hat{\mathbf{\Lambda}}_{0}\right)+\varepsilon^{1 / 2} E_{0} \Lambda_{0}^{3} F(\mathbf{Q})+o\left(\varepsilon^{1 / 2}\right) .
$$

Then the assumption $K_{\varepsilon}(\boldsymbol{\Lambda}, \mathbf{Q})=c$, together with 6.8, implies that $F(\mathbf{Q})=\hat{F}+o(1)$, a contradiction with Lemma 6.1, provided $\delta$ is chosen small enough. This concludes the proof of (H2).

Proof of Lemma 6.1. We first note the existence of a positive constant $C$ independent of $Q \in \Omega$ such that

$$
\int_{\Omega} \frac{1}{|x-Q|^{3}} d x \leq C .
$$

So the integral term in $F(\mathbf{Q})$ is uniformly bounded in $\delta$.

Let $Q \in \Omega$ be close to $\partial \Omega$, and $Q_{0}$ be the nearest point of $\partial \Omega$ to $Q$. It is easily checked that

$$
H(x, Q)=-\frac{1}{c_{5}\left|x-Q^{*}\right|^{3}}+O\left(\frac{1}{(d(Q, \partial \Omega))^{2}}\right) \quad \text { as } d(Q, \partial \Omega) \rightarrow 0
$$

uniformly in $\Omega$, where $Q^{*}$ is the reflection of $Q$ across the boundary, that is, the symmetric point to $Q$ with respect to $Q_{0}$ (see Appendix B). In particular,

$$
H(Q, Q)=-\frac{1}{8 c_{5}(d(Q, \partial \Omega))^{3}}+O\left(\frac{1}{(d(Q, \partial \Omega))^{2}}\right) .
$$


On the other hand, we have

$$
G\left(Q_{i}, Q_{j}\right)=\frac{1}{c_{5}\left|Q_{i}-Q_{j}\right|^{3}}-H\left(Q_{i}, Q_{j}\right)
$$

Then, in view of 6.5), we see that

$$
\max _{\mathbf{Q} \in \mathcal{M}_{\delta}} F(\mathbf{Q}) \leq-C / \delta^{3} \quad \text { as } \delta \rightarrow 0
$$

where $C$ is some strictly positive constant.

Proof of Theorem 1.1 completed. We proved that for $\varepsilon$ small enough, $I_{\varepsilon}$ has a critical point $\left(\boldsymbol{\Lambda}^{\varepsilon}, \mathbf{Q}^{\varepsilon}\right)$.

Let $u_{\varepsilon}=W_{\boldsymbol{\Lambda}^{\varepsilon}, \overline{\mathbf{Q}}^{\varepsilon}, \varepsilon}+\phi_{\boldsymbol{\Lambda}^{\varepsilon}, \overline{\mathbf{Q}}^{\varepsilon}, \varepsilon}$. Then $u_{\varepsilon}$ is a nontrivial solution to problem (1.12). The strong maximum principle shows that $u_{\varepsilon}>0$ in $\Omega_{\varepsilon}$. Let $u_{\mu}=\varepsilon^{-3 / 2} u_{\varepsilon}(x / \varepsilon)$. By our construction, $u_{\mu}$ has all the properties of Theorem 1.1 .

\section{Appendix A: Proof of Lemma 2.1}

From the definition 2.8 of $W, 2.10$ and 2.5 , we know that

$$
\begin{aligned}
S_{\varepsilon}[W] & =-\Delta W+\varepsilon^{3 / 2} W-15 W_{+}^{7 / 3} \\
& =15 \sum_{j=1}^{K} U_{j}^{7 / 3}+\varepsilon^{5} \sum_{j=1}^{K} \hat{U}_{j}-15\left(\sum_{j=1}^{K}\left(U_{j}+\varepsilon^{5 / 2} \hat{U}_{j}\right)+\eta \varepsilon^{5 / 2}\right)^{7 / 3}+O\left(\varepsilon^{8}\right) \\
& =\varepsilon^{5} \sum_{j=1}^{K} \hat{U}_{j}+O\left[\sum_{i \neq j} U_{j}^{4 / 3}\left(U_{i}+\varepsilon^{5 / 2}\right)+\varepsilon^{10 / 3} \sum_{j=1}^{K} U_{j}+\varepsilon^{35 / 6}\right] .
\end{aligned}
$$

According to the definition of $U_{j}=U_{\Lambda_{j}, Q_{j} / \varepsilon}$ and the fact that in $\mathcal{M}_{\delta}$ the points $Q_{j}$ remain far apart, we have

$$
U_{j}=O\left(\langle z-\overline{\mathbf{Q}}\rangle^{-3}\right), \quad U_{j}^{4 / 3} U_{i}=O\left(\varepsilon^{3}\langle z-\overline{\mathbf{Q}}\rangle^{-4}\right) \quad \text { for } i \neq j .
$$

From 2.3, 2.2) and 2.5, we also have

$$
\hat{U}_{j}=O\left(\langle z-\overline{\mathbf{Q}}\rangle^{-1 / 2}\right)
$$

Combining these facts yields estimate 2.14 . Estimates for $D_{\Lambda} S_{\varepsilon}[W]$ and $D_{\overline{\mathbf{Q}}} S_{\varepsilon}[W]$ are obtained exactly in the same way.

We now turn to the proof of the energy estimate 2.15. From 2.10 and 2.11 we deduce that

$$
\int_{\Omega_{\varepsilon}}|\nabla W|^{2}+\varepsilon^{5 / 2} \int_{\Omega_{\varepsilon}} W^{2}=15 \sum_{j=1}^{K} \int_{\Omega_{\varepsilon}} U_{j}^{7 / 3} W+\varepsilon^{5} \sum_{j=1}^{K} \int_{\Omega_{\varepsilon}} \hat{U}_{j} W+o\left(\varepsilon^{3}\right) .
$$


The definition 2.8 of $W$ and 7.2 yield $\left|W-\eta \varepsilon^{5 / 2}\right|=O\left(\langle z-\overline{\mathbf{Q}}\rangle^{-3}\right)$, whence, in view of (7.2) and (2.2),

$$
\begin{aligned}
\varepsilon^{5} \sum_{j=1}^{K} \int_{\Omega_{\varepsilon}} \hat{U}_{j} W & =\eta \varepsilon^{5 / 2} \varepsilon^{5} \int_{\Omega_{\varepsilon}} \sum_{j=1}^{K}\left(-\Psi_{j}-c_{5} \varepsilon^{1 / 2} \Lambda_{j}^{3 / 2} H\left(\varepsilon z, Q_{j}\right)\right)+o\left(\varepsilon^{3}\right) \\
& =-c_{5} \eta \varepsilon^{3} \sum_{j=1}^{K} \Lambda_{j}^{3 / 2} \int_{\Omega} H\left(x, Q_{j}\right) d x+o\left(\varepsilon^{3}\right) \\
& =-\eta \varepsilon^{3} \sum_{j=1}^{K} \Lambda_{j}^{3 / 2} \int_{\Omega} \frac{1}{\left|x-Q_{j}\right|^{3}} d x+o\left(\varepsilon^{3}\right) .
\end{aligned}
$$

Concerning the first terms on the right hand side of $(7.3)$, we remark that in view of the definitions of $U_{i}, \hat{U}_{i}$ and 2.2 , for $i \neq j$ we have on $B_{j}=B\left(\bar{Q}_{j}, \delta / 2 \varepsilon\right)$,

$$
\left(U_{i}+\varepsilon^{5 / 2} \hat{U}_{i}\right)(z)=\frac{\varepsilon^{3} \Lambda_{i}^{3 / 2}}{\left|Q_{j}-Q_{i}\right|^{3}}-c_{5} \varepsilon^{3} \Lambda_{i}^{3 / 2} H\left(Q_{j}, Q_{i}\right)+O\left(\varepsilon^{4}\left|z-\bar{Q}_{j}\right|+\varepsilon^{7 / 2}\right) .
$$

As $U_{i}+\varepsilon^{5 / 2} \hat{U}_{i}=O\left(\langle z-\overline{\mathbf{Q}}\rangle^{-3}+\varepsilon^{5 / 2}\right)$ and, outside $B_{j}, U_{j}^{7 / 3}=O\left(\varepsilon^{7}\right)$, we obtain, for $i \neq j$,

$$
15 \int_{\Omega_{\varepsilon}} U_{j}^{7 / 3}\left(U_{i}+\varepsilon^{5 / 2} \hat{U}_{i}\right)=c_{5}^{2} \varepsilon^{3} \Lambda_{i}^{3 / 2} \Lambda_{j}^{3 / 2} G\left(Q_{i}, Q_{j}\right)+o\left(\varepsilon^{3}\right),
$$

noticing that

$$
15 \int_{\mathbb{R}^{5}} U_{j}^{7 / 3}=c_{5} \Lambda_{j}^{3 / 2}
$$

In the same way we find, for $i=j$,

$$
\begin{aligned}
15 \int_{\Omega_{\varepsilon}} U_{j}^{7 / 3} & \left(U_{j}+\varepsilon^{5 / 2} \hat{U}_{j}\right) \\
& =15 \int_{\mathbb{R}^{5}} U_{1,0}^{10 / 3}-15 \varepsilon^{5 / 2} \int_{\Omega_{\varepsilon}} U_{j}^{7 / 3} \Psi_{j}-c_{5}^{2} \varepsilon^{3} \Lambda_{j}^{3} H\left(Q_{j}, Q_{j}\right)+o\left(\varepsilon^{3}\right) .
\end{aligned}
$$

Thus we obtain

$$
\begin{aligned}
\int_{\Omega_{\varepsilon}}|\nabla W|^{2}+\varepsilon^{5 / 2} & \int_{\Omega_{\varepsilon}} W^{2}=15 K \int_{\mathbb{R}^{5}} U_{1,0}^{10 / 3}-15 \varepsilon^{5 / 2} \sum_{j=1}^{K} \int_{\mathbb{R}^{5}} U_{j}^{7 / 3} \Psi_{j} \\
& -c_{5}^{2} \varepsilon^{3}\left[\sum_{j=1}^{K} \Lambda_{j}^{3} H\left(Q_{j}, Q_{j}\right)-\sum_{i \neq j} \Lambda_{i}^{3 / 2} \Lambda_{j}^{3 / 2} G\left(Q_{i}, Q_{j}\right)\right] \\
& +\eta \varepsilon^{5 / 2} c_{5} \sum_{j=1}^{K} \Lambda_{j}^{3 / 2}-\eta \varepsilon^{3} \sum_{j=1}^{K} \Lambda_{j}^{3 / 2} \int_{\Omega} \frac{1}{\left|x-Q_{j}\right|^{3}} d x+o\left(\varepsilon^{3}\right) .
\end{aligned}
$$


It only remains to estimate

$$
\begin{aligned}
\int_{\Omega_{\varepsilon}} W_{+}^{10 / 3}= & \int_{\Omega_{\varepsilon}}\left(\sum_{j=1}^{K}\left(U_{j}+\varepsilon^{5 / 2} \hat{U}_{j}\right)+\eta \varepsilon^{5 / 2}\right)_{+}^{10 / 3} \\
= & \int_{\Omega_{\varepsilon}}\left(\sum_{j=1}^{K} U_{j}\right)^{10 / 3}+\frac{10}{3} \varepsilon^{5 / 2} \int_{\Omega_{\varepsilon}}\left(\sum_{j=1}^{K} U_{j}\right)^{7 / 3}\left(\sum_{j=1}^{K} \hat{U}_{j}\right) \\
& +\frac{10}{3} \eta \varepsilon^{5 / 2} \int_{\Omega_{\varepsilon}}\left(\sum_{j=1}^{K} U_{j}\right)^{7 / 3}+O\left(\int_{\Omega_{\varepsilon}}\left(\varepsilon^{5} \sum_{j=1}^{K} U_{j}^{4 / 3}+\varepsilon^{25 / 3}\right)\right) \\
= & \sum_{j=1}^{K} \int_{\Omega_{\varepsilon}} U_{j}^{10 / 3}+\frac{10}{3} \sum_{i \neq j} \int_{\Omega_{\varepsilon}} U_{j}^{7 / 3}\left(U_{i}+\hat{U}_{i}\right)+\frac{10}{3} \varepsilon^{5 / 2} \sum_{j=1}^{K} \int_{\Omega_{\varepsilon}} U_{j}^{7 / 3} \hat{U}_{j} \\
& +\frac{10}{3} \eta \varepsilon^{5 / 2} \sum_{j=1}^{K} \int_{\Omega_{\varepsilon}} U_{j}^{7 / 3}+o\left(\varepsilon^{3}\right)
\end{aligned}
$$

since, as a consequence of the definition of the $U_{j}$ 's and the fact that the $Q_{j}$ 's remain far apart in $\mathcal{M}_{\delta}$ (see for instance (7.1),

$$
\varepsilon^{5} \int_{\Omega_{\varepsilon}} U_{j}^{4 / 3}=O\left(\varepsilon^{4}\right)
$$

and, for $i \neq j$,

$$
\int_{\Omega_{\varepsilon}} U_{j}^{4 / 3} U_{i}=O\left(\varepsilon^{2}\right), \quad \int_{\Omega_{\varepsilon}} U_{j}^{4 / 3} U_{i}^{2}=O\left(\varepsilon^{4}\right) .
$$

Therefore, the same computations as above yield

$$
\begin{aligned}
\int_{\Omega_{\varepsilon}} W_{+}^{10 / 3}= & K \int_{\mathbb{R}^{5}} U_{1,0}^{10 / 3}-\frac{10}{3} \varepsilon^{5 / 2} \sum_{j=1}^{K} \int_{\mathbb{R}^{5}} U_{j}^{7 / 3} \Psi_{j}+\frac{2}{9} \eta \varepsilon^{5 / 2} c_{5} \sum_{j=1}^{K} \Lambda_{j}^{3 / 2} \\
& -\frac{2}{9} c_{5}^{2} \varepsilon^{3}\left[\sum_{j=1}^{K} \Lambda_{j}^{3} H\left(Q_{j}, Q_{j}\right)-\sum_{i \neq j} \Lambda_{i}^{3 / 2} \Lambda_{j}^{3 / 2} G\left(Q_{i}, Q_{j}\right)\right]+o\left(\varepsilon^{3}\right) .
\end{aligned}
$$

Combining this expansion with 7.5 , we obtain

$$
\begin{aligned}
J_{\varepsilon}[W]= & \frac{1}{2} \int_{\Omega_{\varepsilon}}|\nabla W|^{2}+\frac{\varepsilon^{5 / 2}}{2} \int_{\Omega_{\varepsilon}} W^{2}-\frac{9}{2} \int_{\Omega_{\varepsilon}} W^{10 / 3} \\
= & 3 K \int_{\mathbb{R}^{5}} U_{1,0}^{10 / 3}+\frac{15}{2} \varepsilon^{5 / 2} \sum_{j=1}^{K} \int_{\mathbb{R}^{5}} U_{j}^{7 / 3} \Psi_{j}-\frac{1}{2} \eta \varepsilon^{5 / 2} c_{5} \sum_{j=1}^{K} \Lambda_{j}^{3 / 2} \\
& +\frac{1}{2} c_{5}^{2} \varepsilon^{3}\left[\sum_{j=1}^{K} \Lambda_{j}^{3} H\left(Q_{j}, Q_{j}\right)-\sum_{i \neq j} \Lambda_{i}^{3 / 2} \Lambda_{j}^{3 / 2} G\left(Q_{i}, Q_{j}\right)\right] \\
& -\frac{c_{5}}{2|\Omega|} \varepsilon^{3}\left(\sum_{j=1}^{K} \Lambda_{j}^{3 / 2}\right) \sum_{j=1}^{K} \Lambda_{j}^{3 / 2} \int_{\Omega} \frac{1}{\left|x-Q_{j}\right|^{3}} d x+o\left(\varepsilon^{3}\right) .
\end{aligned}
$$


Lastly, we notice that in view of (2.1),

$$
15 \int_{\mathbb{R}^{5}} U_{j}^{7 / 3} \Psi_{j}=\int_{\mathbb{R}^{5}} U_{j}^{2}=\left(\int_{\mathbb{R}^{5}} U_{1,0}^{2}\right) \Lambda_{j}^{2}=\frac{c_{5} \pi}{16} \Lambda_{j}^{2},
$$

whence, according to the definition 2.9) of $\eta$,

$$
15 \sum_{j=1}^{K} \int_{\mathbb{R}^{5}} U_{j}^{7 / 3} \Psi_{j}-\eta c_{5} \sum_{j=1}^{K} \Lambda_{j}^{3 / 2}=\frac{c_{5} \pi}{16} \sum_{j=1}^{K} \Lambda_{j}^{2}-\frac{c_{5}^{2}}{|\Omega|}\left(\sum_{j=1}^{K} \Lambda_{j}^{3 / 2}\right)^{2} .
$$

Finally, we obtain

$$
\begin{aligned}
J_{\varepsilon}[W] & =A_{0}-\varepsilon^{5 / 2} D_{0}\left(\sum_{j=1}^{K} \Lambda_{j}^{3 / 2}\right)^{2}+\varepsilon^{5 / 2} B_{0} \sum_{j=1}^{K} \Lambda_{j}^{2}+\varepsilon^{3} E_{0}\left[\sum_{j=1}^{K} \Lambda_{j}^{3} H\left(Q_{j}, Q_{j}\right)\right. \\
& \left.-\sum_{i \neq j} \Lambda_{i}^{3 / 2} \Lambda_{j}^{3 / 2} G\left(Q_{i}, Q_{j}\right)-F_{0}\left(\sum_{j=1}^{K} \Lambda_{j}^{3 / 2}\right) \sum_{j=1}^{K} \Lambda_{j}^{3 / 2} \int_{\Omega} \frac{1}{\left|x-Q_{j}\right|^{3}} d x\right]+o\left(\varepsilon^{3}\right)
\end{aligned}
$$

where $A_{0}, B_{0}, D_{0}, E_{0}, F_{0}>0$ are all generic constants which can be traced back from the computations, namely:

$$
A_{0}=\frac{3 \pi c_{5}}{256}, \quad B_{0}=\frac{\pi c_{5}}{32}, \quad D_{0}=\frac{c_{5}^{2}}{2|\Omega|}, \quad E_{0}=\frac{c_{5}^{2}}{2}, \quad F_{0}=\frac{1}{c_{5}|\Omega|} .
$$

To prove estimate 2.16, we observe that

$$
D_{\Lambda_{j}} J_{\varepsilon}[W]=\int_{\Omega} S_{\varepsilon}[W] \partial_{\Lambda_{j}} W=\int_{\Omega} S_{\varepsilon}[W] \partial_{\Lambda_{j}}\left(U_{j}+\varepsilon^{5 / 2} \hat{U}_{j}+\eta \varepsilon^{5 / 2}\right)+O\left(\varepsilon^{3}\right) .
$$

Then the rest of the proof is similar to the previous one. (Note that here we just need an error in $O\left(\varepsilon^{3}\right)$.)

\section{Appendix B: Proof of Lemma 3.1}

To prove (3.6), we show that there exists a constant $C$, independent of $x$ and $y$, such that

$$
|G(x, y)| \leq \frac{C}{|x-y|^{3}} .
$$

We recall the decomposition of $G$ :

$$
G(x, y)=K(|x-y|)-H(x, y)
$$

where $K(|x-y|)$ is the singular part of $G$ and $H(x, y)$ is the regular part. Since $|K(|x-y|)|=\frac{1}{c_{5}|x-y|^{3}}$, it remains to show that

$$
|H(x, y)| \leq \frac{C}{|x-y|^{3}}
$$


Note that if for some fixed $d_{0}>0, d(x, \partial \Omega)>d_{0}$ or $d(y, \partial \Omega)>d_{0}$, then $|H(x, y)| \leq C$ and $(8.1)$ holds. So we just need to estimate $H(x, y)$ for $d(x, \partial \Omega)$ and $d(y, \partial \Omega)$ small. For $y \in \Omega$ such that $d=d(y, \partial \Omega)$ is sufficiently small, there exists a unique point $\bar{y} \in \partial \Omega$ such that $d=|y-\bar{y}|$. Let $y^{*}$ be the reflection point of $y$ through the boundary, i.e. $y^{*}-y=2(\bar{y}-y)$, and consider the auxiliary function

$$
H^{*}(x, y)=K\left(\left|x-y^{*}\right|\right) .
$$

Then $H^{*}$ satisfies $\Delta H^{*}=0$ in $\Omega$ and, on $\partial \Omega$,

$$
\frac{\partial}{\partial v}\left(H^{*}(x, y)\right)=-\frac{\partial}{\partial v}(K(|x-y|))+O\left(\frac{1}{d^{2}}\right) .
$$

Since both $\overline{K(|x-y|)}$ and $\overline{K\left(\left|x-y^{*}\right|\right)}$ are uniformly bounded, we derive that

$$
H(x, y)=-H^{*}(x, y)+O\left(\frac{1}{d^{2}}\right),
$$

which proves (8.1) for $x, y \in \Omega$. This implies, for $x \in \Omega$,

$$
|u(x)| \leq C \int_{\Omega} \frac{|f(y)|}{|x-y|^{3}} d y .
$$

If $x \in \partial \Omega$, we consider a sequence of points $x_{i} \in \Omega$ with $x_{i} \rightarrow x \in \partial \Omega$ and take the limit in 8.2 . Lebesgue's Dominated Convergence Theorem applies and $(3.6)$ is proved.

Acknowledgments. The research of the second author is supported by an Earmarked Grant from RGC of Hong Kong and a direct grant from CUHK.

\section{References}

[1] Adimurthi, Mancini, G.: The Neumann problem for elliptic equations with critical nonlinearity. In: A Tribute in Honour of G. Prodi, Scuola Norm. Sup. Pisa, 9-25 (1991) Zbl 0836.35048 MR 1205370

[2] Adimurthi, Mancini, G.: Geometry and topology of the boundary in the critical Neumann problem. J. Reine Angew. Math. 456, 1-18 (1994) Zbl 0804.35036 MR 1301449

[3] Adimurthi, Pacella, F., Yadava, S. L.: Interaction between the geometry of the boundary and positive solutions of a semilinear Neumann problem with critical nonlinearity. J. Funct. Anal. 113, 318-350 (1993) Zbl 0793.35033 MR 1218099

[4] Adimurthi, Yadava, S. L.: On a conjecture of Lin-Ni for a semilinear Neumann problem. Trans. Amer. Math. Soc. 336, 631-637 (1993) Zbl 0787.35030 MR 1156299

[5] Adimurthi, Yadava, S. L.: Existence and nonexistence of positive radial solutions of Neumann problems with critical Sobolev exponents. Arch. Rat. Mech. Anal. 115, 275-296 (1991) Zbl 0839.35041 MR 1106295

[6] Adimurthi, Yadava, S. L.: Nonexistence of positive radial solutions of a quasilinear Neumann problem with a critical Sobolev exponent. Arch. Rat. Mech. Anal. 139, 239-253 (1997) Zbl 0910.35050 MR 1480241 
[7] Bates, P., Dancer, E. N., Shi, J.: Multi-spike stationary solutions of the Cahn-Hilliard equation in higher-dimension and instability. Adv. Differential Equations 4, 1-69 (1999) Zbl pre01493588 MR 1667283

[8] Bates, P., Fusco, G.: Equilibria with many nuclei for the Cahn-Hilliard equation. J. Differential Equations 160, 283-356 (2000) Zbl 0990.35016 MR 1737000

[9] Brezis, H., Nirenberg, L.: Positive solutions of nonlinear elliptic equations involving critical Sobolev exponents. Comm. Pure Appl. Math. 36, 437-477 (1983) Zbl 0541.35029 MR 0709644

[10] Budd, C., Knapp, M., Peletier, L.: Asymptotic behavior of solutions of elliptic equations with critical exponent and Neumann boundary conditions. Proc. Roy. Soc. Edinburgh 117, 225-250 (1991) Zbl 0733.35038 MR 1103293

[11] Caffarelli, L., Gidas, B., Spruck, J.: Asymptotic symmetry and local behavior of semilinear elliptic equations with critical Sobolev growth. Comm. Pure Appl. Math. 42 (1989), 271-297 Zbl 0702.35085 MR 0982351

[12] Cerami, G., Wei, J.: Multiplicity of multiple interior peaks solutions for some singularly perturbed Neumann problems. Int. Math. Res. Not. 1998, no. 12, 601-626 Zbl 0916.35037 MR 1635869

[13] Dancer, E. N., Yan, S.: Multipeak solutions for a singularly perturbed Neumann problem. Pacific J. Math. 189, 241-262 (1999) Zbl 0933.35070 MR 1696122

[14] Dancer, E. N., Yan, S.: Interior and boundary peak solutions for a mixed boundary value problem. Indiana Univ. Math. J. 48, 1177-1212 (1999) Zbl 0948.35055

[15] Ghoussoub, N., Gui, C.: Multi-peak solutions for a semilinear Neumann problem involving the critical Sobolev exponent. Math. Z. 229, 443-474 (1998) Zbl 0955.35024 MR 1658569

[16] Ghoussoub, N., Gui, C., Zhu, M.: On a singularly perturbed Neumann problem with the critical exponent. Comm. Partial Differential Equations 26, 1929-1946 (2001) Zbl 0997.35021 MR 1876408

[17] Gierer, A., Meinhardt, H.: A theory of biological pattern formation. Kybernetik (Berlin) 12, 30-39 (1972)

[18] Grossi, M., Pistoia, A.: On the effect of critical points of distance function in superlinear elliptic problems. Adv. Differential Equations 5, 1397-1420 (2000) Zbl 0989.35054 MR 1785679

[19] Grossi, M., Pistoia, A., Wei, J.: Existence of multipeak solutions for a semilinear elliptic problem via nonsmooth critical point theory. Calc. Var. Partial Differential Equations 11, 143-175 (2000) Zbl 0964.35047 MR 1782991

[20] Gui, C.: Multi-peak solutions for a semilinear Neumann problem. Duke Math. J. 84, 739-769 (1996)

[21] Gui, C., Lin, C. S.: Estimates for boundary-bubbling solutions to an elliptic Neumann problem. J. Reine Angew. Math. 546, 201-235 (2002) Zbl pre01738273 MR 1900999

[22] Gui, C., Wei, J.: Multiple interior peak solutions for some singularly perturbed Neumann problems. J. Differential Equations 158, 1-27 (1999) Zbl pre01384457 MR 1721719

[23] Gui, C., Wei, J.: On multiple mixed interior and boundary peak solutions for some singularly perturbed Neumann problems. Canad. J. Math. 52, 522-538 (2000) Zbl 0949.35052 MR 1758231

[24] Gui, C., Wei, J., Winter, M.: Multiple boundary peak solutions for some singularly perturbed Neumann problems. Ann. Inst. H. Poincaré Anal. Non Linéaire 17, 47-82 (2000) Zbl 0944.35020 MR 1743431

[25] Khenissy, S., Rey, O.: A criterion for existence of solutions to the supercritical Bahri-Coron's problem. Houston J. Math. 30, 587-613 (2004) Zbl pre02115763 MR 2084920 
[26] Li, Y. Y.: On a singularly perturbed equation with Neumann boundary condition. Comm. Partial Differential Equations 23, 487-545 (1998) Zbl 0898.35004 MR 1620632

[27] Li, Y. Y., Zhu, M.: Yamabe type equations on three-dimensional Riemannian manifolds. Comm. Contemp. Math. 1, 1-50 (1999) Zbl 0973.53029 MR 1681811

[28] Lin, C. S., Ni, W. M.: On the diffusion coefficient of a semilinear Neumann problem. In: Lecture Notes in Math. 1340, Springer, Berlin, 160-174 (1986) Zbl 0704.35050 MR 0974610

[29] Lin, C. S., Ni, W. M., Takagi, I.: Large amplitude stationary solutions to a chemotaxis system. J. Differential Equations 72, 1-27 (1988) Zbl 0676.35030 MR 0929196

[30] Maier-Paape, S., Schmitt, K., Wang, Z. Q.: On Neumann problems for semilinear elliptic equations with critical nonlinearity: existence and symmetry of multi-peaked solutions. Comm. Part. Differential Equations 22, 1493-1527 (1997) Zbl 0895.35040 MR 1469580

[31] Musso, M., Pistoia, A.: Multispike solutions for a nonlinear elliptic problem involving the critical Sobolev exponent. Indiana Univ. Math. J. 51, 541-579 (2002) Zbl pre01990941 MR 1911045

[32] Ni, W. M.: Diffusion, cross-diffusion, and their spike-layer steady states. Notices Amer. Math. Soc. 45, 9-18 (1998) Zbl 0917.35047 MR 1490535

[33] Ni, W. M., Pan, X. B., Takagi, I.: Singular behavior of least-energy solutions of a semilinear Neumann problem involving critical Sobolev exponents. Duke Math. J. 67, 1-20 (1992) Zbl 0785.35041 MR 1174600

[34] Ni, W. M., Takagi, I.: On the shape of least-energy solutions to a semilinear Neumann problem. Comm. Pure Appl. Math. 44, 819-851 (1991) Zbl 0754.35042 MR 1115095

[35] Ni, W. M., Takagi, I.: Locating the peaks of least-energy solutions to a semilinear Neumann problem. Duke Math. J. 70, 247-281 (1993) Zbl 0796.35056 MR 1219814

[36] del Pino, M., Felmer, P., Musso, M.: Two-bubble solutions in the super-critical Bahri-Coron's problem. Calc. Var. Partial Differential Equations 16, 113-145 (2003) Zbl pre01922405 MR 1956850

[37] del Pino, M., Felmer, P., Wei, J.: On the role of mean curvature in some singularly perturbed Neumann problems. SIAM J. Math. Anal. 31, 63-79 (1999) Zbl 0942.35058 MR 1742305

[38] Rey, O.: The role of the Green's function in a nonlinear elliptic problem involving the critical Sobolev exponent. J. Funct. Anal. 89, 1-52 (1990) Zbl 0786.35059 MR 1040954

[39] Rey, O.: An elliptic Neumann problem with critical nonlinearity in three dimensional domains. Comm. Contemp. Math. 1, 405-449 (1999) Zbl 0954.35065 MR 1707889

[40] Rey, O.: The question of interior blow-up points for an elliptic Neumann problem: the critical case. J. Math. Pures Appl. 81, 655-696 (2002) Zbl pre01975948 MR 1968337

[41] Rey, O., Wei, J.: Blow-up solutions for an elliptic Neumann problem with sub- or supercritical nonlinearity, I: $N=3$. J. Funct. Anal., to appear

[42] Rey, O., Wei, J.: Blow-up solutions for an elliptic Neumann problem with sub- or supercritical nonlinearity, II: $N \geq 4$. Submitted

[43] Wang, X. J.: Neumann problems of semilinear elliptic equations involving critical Sobolev exponents. J. Differential Equations 93, 283-310 (1991) Zbl 0766.35017 MR 1125221

[44] Wang, Z. Q.: The effect of domain geometry on the number of positive solutions of Neumann problems with critical exponents. Differential Integral Equations 8, 1533-1554 (1995) Zbl 0829.35041 MR 1329855

[45] Wang, Z. Q.: High energy and multi-peaked solutions for a nonlinear Neumann problem with critical exponent. Proc. Roy. Soc. Edinburgh Sect. A 125, 1003-1029 (1995) Zbl 0877.35050 MR 1361630

[46] Wang, Z. Q.: Construction of multi-peaked solution for a nonlinear Neumann problem with critical exponent. Nonlinear Anal. 27, 1281-1306 (1996) Zbl 0862.35040 MR 1408871 
[47] Wang, X., Wei, J.: On the equation $\Delta u+K(x) u^{\frac{n+2}{n-2} \pm \varepsilon^{2}}=0$ in $\mathbb{R}^{n}$. Rend. Circ. Mat. Palermo 44, 365-400 (1995) Zbl 0859.35029 MR 1388753

[48] Wei, J.: On the interior spike layer solutions of singularly perturbed semilinear Neumann problems. Tohoku Math. J. 50, 159-178 (1998) Zbl $0918.35024 \mid$ MR 1622042

[49] Wei, J.: On the boundary spike layer solutions of singularly perturbed semilinear Neumann problem. J. Differential Equations 134, 104-133 (1997) Zbl 0873.35007 MR 1429093

[50] Wei, J., Winter, M.: Stationary solutions for the Cahn-Hilliard equation. Ann. Inst. H. Poincaré Anal. Non Linéaire 15, 459-492 (1998) Zbl 0910.35049 MR 1632937

[51] Wei, J., Xu, X.: Uniqueness and a priori estimates for some nonlinear elliptic Neumann equations in $\mathbb{R}^{3}$. Pacific J. Math., to appear

[52] Zhu, M.: Uniqueness results through a priori estimates, I. A three dimensional Neumann problem. J. Differential Equations 154, 284-317 (1999) Zbl 0927.35041 MR 1691074 